\title{
A NEW KIND OF LAX-OLEINIK TYPE OPERATOR WITH PARAMETERS FOR TIME-PERIODIC POSITIVE DEFINITE LAGRANGIAN SYSTEMS
}

\author{
KAIZHI WANG ${ }^{1,2}$ AND JUN YAN ${ }^{1}$
}

\begin{abstract}
In this paper we introduce a new kind of Lax-Oleinik type operator with parameters associated with positive definite Lagrangian systems for both the time-periodic case and the time-independent case. On one hand, the new family of Lax-Oleinik type operators with an arbitrary $u \in C\left(M, \mathbb{R}^{1}\right)$ as initial condition converges to a backward weak KAM solution in the timeperiodic case, while it was shown by Fathi and Mather that there is no such convergence of the Lax-Oleinik semigroup. On the other hand, the new family of Lax-Oleinik type operators with an arbitrary $u \in C\left(M, \mathbb{R}^{1}\right)$ as initial condition converges to a backward weak KAM solution faster than the Lax-Oleinik semigroup in the time-independent case.
\end{abstract}

\section{INTRODUCTION}

Let $M$ be a compact and connected smooth manifold. Denote by $T M$ its tangent bundle and $T^{*} M$ the cotangent one. Consider a $C^{\infty}$ Lagrangian $L: T M \times \mathbb{R}^{1} \rightarrow$ $\mathbb{R}^{1},(x, v, t) \mapsto L(x, v, t)$. We suppose that $L$ satisfies the following conditions introduced by Mather [33]:

(H1) Periodicity. $L$ is 1 -periodic in the $\mathbb{R}^{1}$ factor, i.e., $L(x, v, t)=L(x, v, t+1)$ for all $(x, v, t) \in T M \times \mathbb{R}^{1}$.

(H2) Positive Definiteness. For each $x \in M$ and each $t \in \mathbb{R}^{1}$, the restriction of $L$ to $T_{x} M \times t$ is strictly convex in the sense that its Hessian second derivative is everywhere positive definite.

(H3) Superlinear Growth. $\lim _{\|v\|_{x} \rightarrow+\infty} \frac{L(x, v, t)}{\|v\|_{x}}=+\infty$ uniformly on $x \in M$, $t \in \mathbb{R}^{1}$, where $\|\cdot\|_{x}$ denotes the norm induced by a Riemannian metric on $T_{x} M$. By the compactness of $M$, this condition is independent of the choice of the Riemannian metric.

Date: December 2010.

2000 Mathematics Subject Classification. 37J50.

Key words and phrases. weak KAM theory; new Lax-Oleinik type operators; time-periodic Lagrangians; Hamilton-Jacobi equations. 
(H4) Completeness of the Euler-Lagrange Flow. The maximal solutions of the Euler-Lagrange equation, which in local coordinates is:

$$
\frac{d}{d t} \frac{\partial L}{\partial v}(x, \dot{x}, t)=\frac{\partial L}{\partial x}(x, \dot{x}, t)
$$

are defined on all of $\mathbb{R}^{1}$.

The Euler-Lagrange equation is a second order periodic differential equation on $M$ and generates a flow of diffeomorphisms $\phi_{t}^{L}: T M \times \mathbb{S}^{1} \rightarrow T M \times \mathbb{S}^{1}, t \in \mathbb{R}^{1}$, where $\mathbb{S}^{1}$ denotes the circle $\mathbb{R}^{1} / \mathbb{Z}$, defined by

$$
\phi_{t}^{L}\left(x_{0}, v_{0}, t_{0}\right)=\left(x\left(t+t_{0}\right), \dot{x}\left(t+t_{0}\right),\left(t+t_{0}\right) \bmod 1\right),
$$

where $x: \mathbb{R}^{1} \rightarrow M$ is the maximal solution of the Euler-Lagrange equation with initial conditions $x\left(t_{0}\right)=x_{0}, \dot{x}\left(t_{0}\right)=v_{0}$. The completeness and periodicity conditions grant that this correctly defines a flow on $T M \times \mathbb{S}^{1}$.

We can associate with $L$ a Hamiltonian, as a function on $T^{*} M \times \mathbb{R}^{1}: H(x, p, t)=$ $\sup _{v \in T_{x} M}\left\{\langle p, v\rangle_{x}-L(x, v, t)\right\}$, where $\langle\cdot, \cdot\rangle_{x}$ represents the canonical pairing between the tangent and cotangent space. The corresponding Hamilton-Jacobi equation is

$$
u_{t}+H\left(x, u_{x}, t\right)=c(L),
$$

where $c(L)$ is the Mañé critical value [32] of the Lagrangian $L$. In terms of Mather's $\alpha$ function $c(L)=\alpha(0)$.

In this paper we also consider time-independent Lagrangians on $M$. Let $L_{a}$ : $T M \rightarrow \mathbb{R}^{1},(x, v) \mapsto L_{a}(x, v)$ be a $C^{2}$ Lagrangian satisfying the following two conditions:

(H2') Positive Definiteness. For each $(x, v) \in T M$, the Hessian second derivative $\frac{\partial^{2} L_{a}}{\partial v^{2}}(x, v)$ is positive definite.

(H3') Superlinear Growth. $\lim _{\|v\|_{x} \rightarrow+\infty} \frac{L_{a}(x, v)}{\|v\|_{x}}=+\infty$ uniformly on $x \in M$.

Since $M$ is compact, the Euler-Lagrange flow $\phi_{t}^{L_{a}}$ is complete under the assumptions (H2') and (H3').

For $x \in M, p \in T_{x}^{*} M$, the conjugated Hamiltonian $H_{a}$ of $L_{a}$ is defined by: $H_{a}(x, p)=\sup _{v \in T_{x} M}\left\{\langle p, v\rangle_{x}-L(x, v)\right\}$. The corresponding Hamilton-Jacobi equation is

$$
H_{a}\left(x, u_{x}\right)=c\left(L_{a}\right)
$$

The Lax-Oleinik semigroup (hereinafter referred to as L-O semigroup) [22, 26, 36 ] is well known in several domains, such as PDE, Optimization and Control Theory, Calculus of Variations and Dynamical Systems. In particular, it plays an essential role in the weak KAM theory (see [14, 15, 16, 17] or [19]). 
Let us first recall the definitions of the L-O semigroups associated with $L_{a}$ (timeindependent case) and $L$ (time-periodic case), respectively. For each $u \in C\left(M, \mathbb{R}^{1}\right)$ and each $t \geq 0$, let

$$
T_{t}^{a} u(x)=\inf _{\gamma}\left\{u(\gamma(0))+\int_{0}^{t} L_{a}(\gamma(s), \dot{\gamma}(s)) d s\right\}
$$

for all $x \in M$, and

$$
T_{t} u(x)=\inf _{\gamma}\left\{u(\gamma(0))+\int_{0}^{t} L(\gamma(s), \dot{\gamma}(s), s) d s\right\}
$$

for all $x \in M$, where the infimums are taken among the continuous and piecewise $C^{1}$ paths $\gamma:[0, t] \rightarrow M$ with $\gamma(t)=x$. In view of (1.3) and (1.4), for each $t \geq 0$, $T_{t}^{a}$ and $T_{t}$ are operators from $C\left(M, \mathbb{R}^{1}\right)$ to itself. It is not difficult to check that $\left\{T_{t}^{a}\right\}_{t \geq 0}$ and $\left\{T_{n}\right\}_{n \in \mathbb{N}}$ are one-parameter semigroups of operators, which means $T_{0}^{a}=I$ (unit operator), $T_{t+s}^{a}=T_{t}^{a} \circ T_{s}^{a}, \forall t, s \geq 0$, and $T_{0}=I, T_{n+m}=T_{n} \circ T_{m}$, $\forall n, m \in \mathbb{N}$, where $\mathbb{N}=\{0,1,2, \cdots\} .\left\{T_{t}^{a}\right\}_{t \geq 0}$ and $\left\{T_{n}\right\}_{n \in \mathbb{N}}$ are called the L-O semigroup associated with $L_{a}$ and $L$, respectively.

The L-O semigroup is used to obtain backward weak KAM solutions (viscosity solutions) first by Lions, Papanicolaou and Varadhan [29] on the $n$-torus $\mathbb{T}^{n}$ and later by Fathi 14] for arbitrary compact manifolds. More precisely, for the timeindependent case, Fathi 14 proves that there exists a unique $c_{0} \in \mathbb{R}^{1}\left(c_{0}=c\left(L_{a}\right)\right)$, such that the semigroup $\hat{T}_{t}^{a}: u \rightarrow T_{t}^{a} u+c_{0} t, t \geq 0$ has a fixed point $u^{*} \in C\left(M, \mathbb{R}^{1}\right)$ and that any fixed point is a backward weak KAM solution of (1.2). In the particular case $M=\mathbb{T}^{n}$, the backward weak KAM solution obtained by Fathi is just the viscosity solution obtained earlier by Lions, Papanicolaou and Varadhan. Moreover, Fathi points out that the above results for the time-independent case are still correct for the time-periodic dependent case [19]. Furthermore, for the timeindependent case, he shows in 17 that for every $u \in C\left(M, \mathbb{R}^{1}\right)$, the uniform limit $\lim _{t \rightarrow+\infty} \hat{T}_{t}^{a} u=\bar{u}$ exists and is a fixed point of $\left\{\hat{T}_{t}^{a}\right\}_{t \geq 0}$, i.e., $\bar{u}$ is a backward weak KAM solution of (1.2). In the same paper Fathi raises the question as to whether the analogous result holds in the time-periodic case. This would be the convergence of $T_{n} u+n c(L), \forall u \in C\left(M, \mathbb{R}^{1}\right)$, as $n \rightarrow+\infty, n \in \mathbb{N}$. In view of the relation between $T_{n}$ and the Peierls barrier $h$ (see [34 or [18, 3, 9]), if the liminf in the definition of the Peierls barrier is not a limit, then the L-O semigroup in the time-periodic case does not converge. Fathi and Mather [18] construct examples where the liminf in the definition of the Peierls barrier is not a limit, thus answering the above question negatively.

The main aim of the present paper is to introduce a new kind of Lax-Oleinik type operator with parameters (hereinafter referred to as new L-O operator) associated with positive definite Lagrangian systems for both the time-periodic case 
and the time-independent case. The new L-O operator associated with the timeindependent Lagrangian is a special case of the one associated with the timeperiodic Lagrangian. We show that

- for the time-periodic Lagrangian $L$, the new family of L-O operators with an arbitrary continuous function on $M$ as initial condition converges to a backward weak KAM solution of (1.1).

- for the time-independent Lagrangian $L_{a}$, the new family of L-O operators is a one-parameter semigroup of operators, and the new L-O semigroup with an arbitrary continuous function on $M$ as initial condition converges to a backward weak KAM solution of (1.2) faster than the L-O semigroup.

Without loss of generality, we will from now on always assume $c\left(L_{a}\right)=c(L)=0$. We view the unit circle $\mathbb{S}^{1}$ as the fundmental domain in $\mathbb{R}^{1}:[0,1]$ with two endpoints identified.

We are now in a position to introduce the new L-O operators mentioned above associated with $L$ and $L_{a}$, respectively.

1.1. Time-periodic case. For each $n \in \mathbb{N}$ and each $u \in C\left(M, \mathbb{R}^{1}\right)$, let

$$
\tilde{T}_{n} u(x)=\inf _{\substack{k \in \mathbb{N} \\ n \leq k \leq 2 n}} \inf _{\gamma}\left\{u(\gamma(0))+\int_{0}^{k} L(\gamma(s), \dot{\gamma}(s), s) d s\right\}
$$

for all $x \in M$, where the second infimum is taken among the continuous and piecewise $C^{1}$ paths $\gamma:[0, k] \rightarrow M$ with $\gamma(k)=x$. One can easily check that for each $n \in \mathbb{N}, \tilde{T}_{n}$ is an operator from $C\left(M, \mathbb{R}^{1}\right)$ to itself, and that $\left\{\tilde{T}_{n}\right\}_{n \in \mathbb{N}}$ is a semigroup of operators.

Definition 1.1. For each $\tau \in[0,1]$ and each $n \in \mathbb{N}$, let $\tilde{T}_{n}^{\tau}=T_{\tau} \circ \tilde{T}_{n}$. Then for each $u \in C\left(M, \mathbb{R}^{1}\right)$,

$$
\tilde{T}_{n}^{\tau} u(x)=\left(T_{\tau} \circ \tilde{T}_{n} u\right)(x)=\inf _{\substack{k \in \mathbb{N} \\ n \leq k \leq 2 n}} \inf _{\gamma}\left\{u(\gamma(0))+\int_{0}^{\tau+k} L(\gamma(s), \dot{\gamma}(s), s) d s\right\}
$$

for all $x \in M$, where the second infimum is taken among the continuous and piecewise $C^{1}$ paths $\gamma:[0, \tau+k] \rightarrow M$ with $\gamma(\tau+k)=x$.

For each $\tau \in[0,1]$ and each $n \in \mathbb{N}$, since $\tilde{T}_{n}^{\tau}=T_{\tau} \circ \tilde{T}_{n}$ and $T_{\tau}, \tilde{T}_{n}$ are both operators from $C\left(M, \mathbb{R}^{1}\right)$ to itself, then $\tilde{T}_{n}^{\tau}$ is an operator from $C\left(M, \mathbb{R}^{1}\right)$ to itself too. We also provide an alternative direct proof of the continuity of $\tilde{T}_{n}^{\tau} u$ for each $u \in C\left(M, \mathbb{R}^{1}\right)$ in Section 3. We call $\tilde{T}_{n}^{\tau}$ the new L-O operator associated with $L$. Note that for $\tau \in[0,1] \backslash\{0\},\left\{\tilde{T}_{n}^{\tau}\right\}_{n \in \mathbb{N}}$ is not a semigroup of operators, while in the particular case $\tau=0,\left\{\tilde{T}_{n}^{0}\right\}_{n \in \mathbb{N}}=\left\{\tilde{T}_{n}\right\}_{n \in \mathbb{N}}$ is a semigroup of operators as mentioned above. For each $n \in \mathbb{N}$ and each $u \in C\left(M, \mathbb{R}^{1}\right)$, let $U_{n}^{u}(x, \tau)=\tilde{T}_{n}^{\tau} u(x)$ for all $(x, \tau) \in M \times[0,1]$. Then $U_{n}^{u}$ is a continuous function on $M \times[0,1]$.

Now we come to the main result: 
Theorem 1.2. For each $u \in C\left(M, \mathbb{R}^{1}\right)$, the uniform limit $\bar{u}=\lim _{n \rightarrow+\infty} U_{n}^{u}$ exists and

$$
\bar{u}(x, \tau)=\inf _{y \in M}\left(u(y)+h_{0, \tau}(y, x)\right)
$$

for all $(x, \tau) \in M \times \mathbb{S}^{1}$. Furthermore, $\bar{u}$ is a backward weak KAM solution of the Hamilton-Jacobi equation

$$
u_{\tau}+H\left(x, u_{x}, \tau\right)=0 .
$$

Remark 1.3. For the definition of the (extended) Peierls barrier $h$, see [34] or [18, 3, 9. For completeness' sake, we recall the definition in Section 3.

In addition, we discuss the relation among uniform limits $\lim _{n \rightarrow+\infty} U_{n}^{u}$, backward weak KAM solutions and viscosity solutions of (1.5). Let $\bar{u} \in C\left(M \times \mathbb{S}^{1}, \mathbb{R}^{1}\right)$. Then the following three statements are equivalent.

- There exists $u \in C\left(M, \mathbb{R}^{1}\right)$ such that the uniform $\operatorname{limit}_{\lim } \lim _{n \rightarrow \infty} U_{n}^{u}=\bar{u}$.

- $\bar{u}$ is a backward weak KAM solution of (1.5).

- $\bar{u}$ is a viscosity solution of (1.5).

See Propositions 3.10, 3.12 for details.

1.2. Time-independent case. Just like the time-periodic case, for each $n \in \mathbb{N}$ and each $u \in C\left(M, \mathbb{R}^{1}\right)$, let

$$
\tilde{T}_{n}^{a} u(x)=\inf _{\substack{k \in \mathbb{N} \\ n \leq k \leq 2 n}} \inf _{\gamma}\left\{u(\gamma(0))+\int_{0}^{k} L_{a}(\gamma(s), \dot{\gamma}(s)) d s\right\}
$$

for all $x \in M$, where the second infimum is taken among the continuous and piecewise $C^{1}$ paths $\gamma:[0, k] \rightarrow M$ with $\gamma(k)=x$. For each $n \in \mathbb{N}, \tilde{T}_{n}^{a}$ is an operator from $C\left(M, \mathbb{R}^{1}\right)$ to itself, and $\left\{\tilde{T}_{n}^{a}\right\}_{n \in \mathbb{N}}$ is a semigroup of operators.

For each $\tau \in[0,1]$ and each $n \in \mathbb{N}$, let $\tilde{T}_{n}^{a, \tau}=T_{\tau}^{a} \circ \tilde{T}_{n}^{a}$. Then for each $u \in$ $C\left(M, \mathbb{R}^{1}\right)$

$$
\tilde{T}_{n}^{a, \tau} u(x)=\left(T_{\tau}^{a} \circ \tilde{T}_{n}^{a} u\right)(x)=\inf _{\substack{k \in \mathbb{N} \\ n \leq k \leq 2 n}} \inf _{\gamma}\left\{u(\gamma(0))+\int_{0}^{\tau+k} L_{a}(\gamma(s), \dot{\gamma}(s)) d s\right\}
$$

for all $x \in M$, where the second infimum is taken among the continuous and piecewise $C^{1}$ paths $\gamma:[0, \tau+k] \rightarrow M$ with $\gamma(\tau+k)=x$. For each $\tau \in[0,1]$ and each $n \in \mathbb{N}, \tilde{T}_{n}^{a, \tau}$ is an operator from $C\left(M, \mathbb{R}^{1}\right)$ to itself. Furthermore, it is not difficult to show that for each $\tau \in[0,1]$ and each $u \in C\left(M, \mathbb{R}^{1}\right)$, the uniform limit $\lim _{n \rightarrow+\infty} \tilde{T}_{n}^{a, \tau} u$ exists and $\lim _{n \rightarrow+\infty} \tilde{T}_{n}^{a, \tau} u=\lim _{n \rightarrow+\infty} T_{n}^{a} u=\bar{u}$, which is a backward weak KAM solution of (1.2), see Remark4.6. It means that the parameter $\tau$ does not effect the convergence of $\left\{\tilde{T}_{n}^{a, \tau} u\right\}_{n \in \mathbb{N}}$. Therefore, without any loss of generality, we take $\tau=0$ and thus consider the operator $\tilde{T}_{n}^{a, 0}=\tilde{T}_{n}^{a}$. In order to 
compare the new family of L-O operators to the full L-O semigroup $\left\{T_{t}^{a}\right\}_{t \geq 0}$, it is convenient to define the new $L$-O operator associated with $L_{a}$ as follows.

Definition 1.4. For each $u \in C\left(M, \mathbb{R}^{1}\right)$ and each $t \geq 0$, let

$$
\tilde{T}_{t}^{a} u(x)=\inf _{t \leq \sigma \leq 2 t} \inf _{\gamma}\left\{u(\gamma(0))+\int_{0}^{\sigma} L_{a}(\gamma(s), \dot{\gamma}(s)) d s\right\}
$$

for all $x \in M$, where the second infimum is taken among the continuous and piecewise $C^{1}$ paths $\gamma:[0, \sigma] \rightarrow M$ with $\gamma(\sigma)=x$.

It is easy to check that $\left\{\tilde{T}_{t}^{a}\right\}_{t \geq 0}: C\left(M, \mathbb{R}^{1}\right) \rightarrow C\left(M, \mathbb{R}^{1}\right)$ is a one-parameter semigroup of operators. We call it the new $L$-O semigroup associated with $L_{a}$. We show that $u \in C\left(M, \mathbb{R}^{1}\right)$ is a fixed point of $\left\{\tilde{T}_{t}^{a}\right\}_{t \geq 0}$ if and only if it is a fixed point of $\left\{T_{t}^{a}\right\}_{t \geq 0}$, and that for each $u \in C\left(M, \mathbb{R}^{1}\right)$, the uniform limit $\lim _{t \rightarrow+\infty} \tilde{T}_{t}^{a} u=$ $\lim _{t \rightarrow+\infty} T_{t}^{a} u=\bar{u}$. For more properties of $\tilde{T}_{t}^{a}$ we refer to Section 4 .

How fast does the L-O semigroup converge? It is an interesting question which is well worth discussing. We believe that there is a deep relation between dynamical properties of Mather sets (or Aubry sets) and the rate of convergence of the LO semigroup. To the best of our knowledge there are now two relative results: In [23], Iturriaga and Sánchez-Morgado prove that if the Aubry set consists in a finite number of hyperbolic periodic orbits or hyperbolic fixed points, the L-O semigroup converges exponentially. Recently, in 38] the authors deal with the rate of convergence problem when the Mather set consists of degenerate fixed points. More precisely, consider the standard Lagrangian in classical mechanics $L_{a}^{0}(x, v)=$ $\frac{1}{2} v^{2}+U(x), x \in \mathbb{S}^{1}, v \in \mathbb{R}^{1}$, where $U$ is a real analytic function on $\mathbb{S}^{1}$ and has a unique global minimum point $x_{0}$. Without loss of generality, one may assume $x_{0}=0, U(0)=0$. Then $c\left(L_{a}^{0}\right)=0$ and $\tilde{\mathcal{M}}_{0}=\{(0,0)\}$, where $\tilde{\mathcal{M}}_{0}$ is the Mather set with cohomology class 0 33. An upper bound estimate of the rate of convergence of the L-O semigroup is provided in 38 . under the assumption that $\{(0,0)\}$ is a degenerate fixed point: for every $u \in C\left(\mathbb{S}^{1}, \mathbb{R}^{1}\right)$, there exists a constant $C>0$ such that

$$
\left\|T_{t}^{a} u-\bar{u}\right\|_{\infty} \leq \frac{C}{\sqrt[k-1]{t}}, \quad \forall t>0,
$$

where $k \in \mathbb{N}, k \geq 2$ depends only on the degree of degeneracy of the minimum point of the potential function $U$.

Naturally, we also care the problem of the rate of convergence of the new L-O semigroup. We compare the rate of convergence of the new L-O semigroup to the rate for the L-O semigroup as follows. First, we show that for each $u \in C\left(M, \mathbb{R}^{1}\right)$, $\left\|\tilde{T}_{t}^{a} u-\bar{u}\right\|_{\infty} \leq\left\|T_{t}^{a} u-\bar{u}\right\|_{\infty}, \forall t \geq 0$. It means that the new L-O semigroup converges faster than the L-O semigroup.

Then, in particular, we consider a class of $C^{2}$ positive definite and superlinear Lagrangians on $\mathbb{T}^{n}$ 


$$
L_{a}^{1}(x, v)=\frac{1}{2}\langle A(x)(v-\omega),(v-\omega)\rangle+f(x, v-\omega), \quad x \in \mathbb{T}^{n}, v \in \mathbb{R}^{n},
$$

where $A(x)$ is an $n \times n$ matrix, $\omega \in \mathbb{S}^{n-1}$ is a given vector, and $f(x, v-\omega)=O(\| v-$ $\left.\omega \|^{3}\right)$ as $v-\omega \rightarrow 0$. It is clear that $c\left(L_{a}^{1}\right)=0$ and $\tilde{\mathcal{M}}_{0}=\tilde{\mathcal{A}}_{0}=\tilde{\mathcal{N}}_{0}=\cup_{x \in \mathbb{T}^{n}}(x, \omega)$, which is a quasi-periodic invariant torus with frequency vector $\omega$ of the EulerLagrange flow associated to $L_{a}^{1}$, where $\tilde{\mathcal{A}}_{0}$ and $\tilde{\mathcal{N}}_{0}$ are the Aubry set and the Mañé set with cohomology class 0 34, respectively. For the Lagrangian system (1.6), we obtain the following two results on the rates of convergence of the L-O semigroup and the new L-O semigroup, respectively.

Theorem 1.5. For each $u \in C\left(\mathbb{T}^{n}, \mathbb{R}^{1}\right)$, there is a constant $K>0$ such that

$$
\left\|T_{t}^{a} u-\bar{u}\right\|_{\infty} \leq \frac{K}{t}, \quad \forall t>0,
$$

where $K$ depends only on $n$ and $u$.

We recall the notations for Diophantine vectors: for $\rho>n-1$ and $\alpha>0$, let

$$
\mathcal{D}(\rho, \alpha)=\left\{\beta \in \mathbb{S}^{n-1}||\langle\beta, k\rangle \mid \geq \frac{\alpha}{|k|^{\rho}}, \forall k \in \mathbb{Z}^{n} \backslash\{0\}\right\},
$$

where $|k|=\sum_{i=1}^{n}\left|k_{i}\right|$.

Theorem 1.6. Given any frequency vector $\omega \in \mathcal{D}(\rho, \alpha)$, for each $u \in C\left(\mathbb{T}^{n}, \mathbb{R}^{1}\right)$, there is a constant $\tilde{K}>0$ such that

$$
\left\|\tilde{T}_{t}^{a} u-\bar{u}\right\|_{\infty} \leq \tilde{K} t^{-\left(1+\frac{4}{2 \rho+n}\right)}, \quad \forall t>0,
$$

where $\tilde{K}$ depends only on $n, \rho, \alpha$ and $u$.

Finally, we construct an example (Example 4.10) to show that the result of Theorem 1.5 is sharp in the sense of order. Therefore, in view of Theorems 1.5 1.6 and Example 4.10, we conclude that the new L-O semigroup converges faster than the L-O semigroup in the sense of order when the Aubry set $\tilde{\mathcal{A}}_{0}$ of the Lagrangian system (1.6) is a quasi-periodic invariant torus with Diophantine frequency vector $\omega \in \mathcal{D}(\rho, \alpha)$.

We hope that the new L-O operator introduced in the present paper will contribute to the development of the Mather theory and the weak KAM theory. At the end of this section, we refer the reader to some good introductory books (lecture notes), survey articles and most recent research articles on the Mather theory and the weak KAM theory: 35, 19, 7, 37, 30, 31, 11, 12, 24, 1, 2, 5, 6, 13, 20, 21.

The rest of the paper is organized as follows. In Section 2 we introduce the basic language and notation used in the sequel. In Section 3 we first study the basic properties of the new L-O operator associated with $L$ and then prove Theorem 1.2. The last part of the section is devoted to the discussion of the relation among 
uniform $\operatorname{limits}_{\lim _{n \rightarrow+\infty}} U_{n}^{u}$, backward weak KAM solutions and viscosity solutions of (1.5). In Section 4 we first study the basic properties of the new L-O semigroup associated with $L_{a}$ and then give the proofs of Theorems 1.5 and 1.6. At last, we construct the example mentioned above (Example 4.10).

\section{NotATiOn AND TERMinOLOGY}

Consider the flat $n$-torus $\mathbb{T}^{n}$, whose universal cover is the Euclidean space $\mathbb{R}^{n}$. We view the torus as a fundamental domain in $\mathbb{R}^{n}$

$$
\bar{A}=\underbrace{[0,1] \times \cdots \times[0,1]}_{n \text { times }}
$$

with opposite faces identified. The unique coordinates $x=\left(x_{1}, \ldots, x_{n}\right)$ of a point in $\mathbb{T}^{n}$ will belong to the half-open cube

$$
A=\underbrace{[0,1) \times \cdots \times[0,1)}_{n \text { times }} .
$$

In these coordinates the standard universal covering projection $\pi: \mathbb{R}^{n} \rightarrow \mathbb{T}^{n}$ takes the form

$$
\pi(\tilde{x})=\left(\left[\tilde{x}_{1}\right], \ldots,\left[\tilde{x}_{n}\right]\right),
$$

where $\left[\tilde{x}_{i}\right]=\tilde{x}_{i} \bmod 1$, denotes the fractional part of $\tilde{x}_{i}\left(\tilde{x}_{i}=\left\{\tilde{x}_{i}\right\}+\left[\tilde{x}_{i}\right]\right.$, where $\left\{\tilde{x}_{i}\right\}$ is the greatest integer not greater than $\tilde{x}_{i}$ ). We can now define operations on $\mathbb{T}^{n}$ using the covering projection: each operation is simply the projection of the usual operation with coordinates in $\mathbb{R}^{n}$. Thus the flat metric $d_{\mathbb{T}^{n}}$ may be defined for any pair of points $x, y \in \mathbb{T}^{n}$ by $d_{\mathbb{T}^{n}}(x, y)=\|x-y\|$, where $\|\cdot\|$ is the usual Euclidean norm on $\mathbb{R}^{n}$. And the distance between points on the torus is at most $\frac{\sqrt{n}}{2}$. For $x \in \mathbb{T}^{n}$ and $R>0, B_{R}(x)=\left\{y \in \mathbb{T}^{n} \mid d_{\mathbb{T}^{n}}(x, y)<R\right\}$ denotes the open ball of the radius $R$ centered on $x$ in $\mathbb{T}^{n}$.

We choose, once and for all, a $C^{\infty}$ Riemannian metric on $M$. It is classical that there is a canonical way to associate to it a Riemannian metric on $T M$. We use the same symbol " $d$ " to denote the distance function defined by the Riemannian metric on $M$ and the distance function defined by the Riemannian metric on $T M$. Denote by $\|\cdot\|_{x}$ the norm induced by the Riemannian metric on the fiber $T_{x} M$ for $x \in M$, and by $\langle\cdot, \cdot\rangle_{x}$ the canonical pairing between $T_{x} M$ and $T_{x}^{*} M$. In particular, for $M=\mathbb{T}^{n}$, we denote $\langle\cdot, \cdot\rangle_{x}$ by $\langle\cdot, \cdot\rangle$ for brevity. We use the same notation $\langle\cdot, \cdot\rangle$ for the standard inner product on $\mathbb{R}^{n}$. However, this should not create any ambiguity.

We equip $C\left(M, \mathbb{R}^{1}\right)$ and $C\left(M \times \mathbb{S}^{1}, \mathbb{R}^{1}\right)$ with the usual uniform topology (the compact-open topology, or the $C^{0}$-topology) defined by the supremum norm $\|\cdot\|_{\infty}$. We use $u \equiv$ const. to denote a constant function whose values do not vary. 


\section{ThE NEW L-O OPERATOR: TIME-PERIODIC CASE}

In this section we first discuss some basic properties of the new L-O operator associated with $L$, i.e., $\left\{\tilde{T}_{n}^{\tau}\right\}$, and then study the uniform convergence of $U_{n}^{u}, \forall u \in$ $C\left(M, \mathbb{R}^{1}\right)$, as $n \rightarrow+\infty$. At last, we discuss the relation among uniform limits $\lim _{n \rightarrow+\infty} U_{n}^{u}$, backward weak KAM solutions and viscosity solutions of (1.5).

3.1. Basic properties of the new L-O operator. Recall the definition of the new L-O operator associated with $L$. For each $\tau \in[0,1]$, each $n \in \mathbb{N}$ and each $u \in C\left(M, \mathbb{R}^{1}\right)$,

$$
\tilde{T}_{n}^{\tau} u(x)=\inf _{\substack{k \in \mathbb{N} \\ n \leq k \leq 2 n}} \inf _{\gamma}\left\{u(\gamma(0))+\int_{0}^{\tau+k} L(\gamma(s), \dot{\gamma}(s), s) d s\right\}
$$

for all $x \in M$, where the second infimum is taken among the continuous and piecewise $C^{1}$ paths $\gamma:[0, \tau+k] \rightarrow M$ with $\gamma(\tau+k)=x$.

First of all, we show that for each $\tau \in[0,1]$ and each $n \in \mathbb{N}, \tilde{T}_{n}^{\tau}$ is an operator from $C\left(M, \mathbb{R}^{1}\right)$ to itself. For this, noticing that $\tilde{T}_{n}^{\tau} u(x) \in \mathbb{R}^{1}$ for all $x \in M$, we only need to prove the following result.

Proposition 3.1. For each $\tau \in[0,1]$, each $n \in \mathbb{N}$ and each $u \in C\left(M, \mathbb{R}^{1}\right), \tilde{T}_{n}^{\tau} u$ is a continuous function on $M$.

Proof. Following Mather ([34], also see [3]), it is convenient to introduce, for $t^{\prime} \geq t$ and $x, y \in M$, the following quantity:

$$
F_{t, t^{\prime}}(x, y)=\inf _{\gamma} \int_{t}^{t^{\prime}} L(\gamma(s), \dot{\gamma}(s), s) d s,
$$

where the infimum is taken over the continuous and piecewise $C^{1}$ paths $\gamma:\left[t, t^{\prime}\right] \rightarrow$ $M$ such that $\gamma(t)=x$ and $\gamma\left(t^{\prime}\right)=y$.

By the definition of $\tilde{T}_{n}^{\tau}$, for each $u \in C\left(M, \mathbb{R}^{1}\right)$ and each $x \in M$, we have

$$
\tilde{T}_{n}^{\tau} u(x)=\inf _{\substack{k \in \mathbb{N} \\ n \leq k \leq 2 n}} \inf _{y \in M}\left(u(y)+F_{0, \tau+k}(y, x)\right) .
$$

Since the function $(y, x) \mapsto F_{0, \tau+k}(y, x)$ is continuous for each $n \leq k \leq 2 n, k \in$ $\mathbb{N}$ (see [3]), then from the compactness of $M$ the function $x \mapsto \inf _{y \in M}(u(y)+$ $\left.F_{0, \tau+k}(y, x)\right)$ is also continuous for each $n \leq k \leq 2 n, k \in \mathbb{N}$. Therefore, the function $x \mapsto \tilde{T}_{n}^{\tau} u(x)$ is continuous on $M$.

Proposition 3.2. For given $\tau \in[0,1], n \in \mathbb{N}, u \in C\left(M, \mathbb{R}^{1}\right)$ and $x \in M$, there exist $n \leq k_{0} \leq 2 n, k_{0} \in \mathbb{N}$ and a minimizing extremal curve $\gamma:\left[0, \tau+k_{0}\right] \rightarrow M$ such that $\gamma\left(\tau+k_{0}\right)=x$ and

$$
\tilde{T}_{n}^{\tau} u(x)=u(\gamma(0))+\int_{0}^{\tau+k_{0}} L(\gamma(s), \dot{\gamma}(s), s) d s .
$$


Proof. Recall that

$$
\tilde{T}_{n}^{\tau} u(x)=\inf _{\substack{k \in \mathbb{N} \\ n \leq k \leq 2 n}} \inf _{y \in M}\left(u(y)+F_{0, \tau+k}(y, x)\right) .
$$

For each $k$, the function $y \mapsto u(y)+F_{0, \tau+k}(y, x)$ is continuous on $M$. Thus, from the compactness of $M$ there exist $y^{k} \in M$ such that

$$
\tilde{T}_{n}^{\tau} u(x)=\inf _{\substack{k \in \mathbb{N} \\ n \leq k \leq 2 n}}\left(u\left(y^{k}\right)+F_{0, \tau+k}\left(y^{k}, x\right)\right) .
$$

Then it is clear that there is $n \leq k_{0} \leq 2 n, k_{0} \in \mathbb{N}$ such that

$$
\tilde{T}_{n}^{\tau} u(x)=u\left(y^{k_{0}}\right)+F_{0, \tau+k_{0}}\left(y^{k_{0}}, x\right) .
$$

It follows from Tonelli's theorem (see, for example, [33]) that there exists a minimizing extremal curve $\gamma:\left[0, \tau+k_{0}\right] \rightarrow M$ such that $\gamma(0)=y^{k_{0}}, \gamma\left(\tau+k_{0}\right)=x$ and

$$
F_{0, \tau+k_{0}}\left(y^{k_{0}}, x\right)=\int_{0}^{\tau+k_{0}} L(\gamma(s), \dot{\gamma}(s), s) d s
$$

Hence,

$$
\tilde{T}_{n}^{\tau} u(x)=u(\gamma(0))+\int_{0}^{\tau+k_{0}} L(\gamma(s), \dot{\gamma}(s), s) d s
$$

\section{Proposition 3.3.}

(1) For $u, v \in C\left(M, \mathbb{R}^{1}\right)$, if $u \leq v$, then $\tilde{T}_{n}^{\tau} u \leq \tilde{T}_{n}^{\tau} v, \forall \tau \in[0,1], \forall n \in \mathbb{N}$.

(2) If $c$ is a constant and $u \in C\left(M, \mathbb{R}^{1}\right)$, then $\tilde{T}_{n}^{\tau}(u+c)=\tilde{T}_{n}^{\tau} u+c, \forall \tau \in[0,1]$, $\forall n \in \mathbb{N}$.

(3) For each $u, v \in C\left(M, \mathbb{R}^{1}\right),\left\|\tilde{T}_{n}^{\tau} u-\tilde{T}_{n}^{\tau} v\right\|_{\infty} \leq\|u-v\|_{\infty}, \forall \tau \in[0,1], \forall n \in \mathbb{N}$.

Proof. For each $\tau \in[0,1]$, each $n \in \mathbb{N}$ and each $x \in M$,

$$
\begin{aligned}
\tilde{T}_{n}^{\tau} u(x) & =\inf _{\substack{k \in \mathbb{N} \\
n \leq k \leq 2 n}} \inf _{y \in M}\left(u(y)+F_{0, \tau+k}(y, x)\right) \\
& \leq \inf _{\substack{k \in \mathbb{N} \\
n \leq k \leq 2 n}} \inf _{y \in M}\left(v(y)+F_{0, \tau+k}(y, x)\right) \\
& =\tilde{T}_{n}^{\tau} v(x),
\end{aligned}
$$

which proves (1). (2) results from the definition of $\tilde{T}_{n}^{\tau}$ directly. To prove (3), we notice that for each $x \in M$,

$$
-\|u-v\|_{\infty}+v(x) \leq u(x) \leq\|u-v\|_{\infty}+v(x) .
$$


From (1) and (2), for each $x \in M$ we have

$$
\tilde{T}_{n}^{\tau} v(x)-\|u-v\|_{\infty} \leq \tilde{T}_{n}^{\tau} u(x) \leq \tilde{T}_{n}^{\tau} v(x)+\|u-v\|_{\infty}, \quad \forall \tau \in[0,1], \forall n \in \mathbb{N} .
$$

Hence, $\left\|\tilde{T}_{n}^{\tau} u-\tilde{T}_{n}^{\tau} v\right\|_{\infty} \leq\|u-v\|_{\infty}, \forall \tau \in[0,1], \forall n \in \mathbb{N}$.

3.2. Uniform convergence of $U_{n}^{u}$. Here we deal with the uniform convergence of $U_{n}^{u}, \forall u \in C\left(M, \mathbb{R}^{1}\right)$, as $n \rightarrow+\infty$. We show that for each $u \in C\left(M, \mathbb{R}^{1}\right)$ the uniform limit $\bar{u}=\lim _{n \rightarrow+\infty} U_{n}^{u}$ exists and

$$
\bar{u}(x, \tau)=\inf _{y \in M}\left(u(y)+h_{0, \tau}(y, x)\right)
$$

for all $(x, \tau) \in M \times \mathbb{S}^{1}$. This is an immediate consequence of Proposition 3.5 below.

Following Mañé 32] and Mather [34, define the action potential and the extended Peierls barrier as follows.

Action Potential: for each $\left(\tau, \tau^{\prime}\right) \in \mathbb{S}^{1} \times \mathbb{S}^{1}$, let

$$
\Phi_{\tau, \tau^{\prime}}\left(x, x^{\prime}\right)=\inf F_{t, t^{\prime}}\left(x, x^{\prime}\right)
$$

for all $\left(x, x^{\prime}\right) \in M \times M$, where the infimum is taken on the set of $\left(t, t^{\prime}\right) \in \mathbb{R}^{2}$ such that $\tau=[t], \tau^{\prime}=\left[t^{\prime}\right]$ and $t^{\prime} \geq t+1$.

Extended Peierls Barrier: for each $\left(\tau, \tau^{\prime}\right) \in \mathbb{S}^{1} \times \mathbb{S}^{1}$, let

$$
h_{\tau, \tau^{\prime}}\left(x, x^{\prime}\right)=\liminf _{t^{\prime}-t \rightarrow+\infty} F_{t, t^{\prime}}\left(x, x^{\prime}\right)
$$

for all $\left(x, x^{\prime}\right) \in M \times M$, where the liminf is restricted to the set of $\left(t, t^{\prime}\right) \in \mathbb{R}^{2}$ such that $\tau=[t], \tau^{\prime}=\left[t^{\prime}\right]$.

From the above definitions, it is not hard to see that

$$
\Phi_{\tau, \tau^{\prime}}\left(x, x^{\prime}\right) \leq h_{\tau, \tau^{\prime}}\left(x, x^{\prime}\right), \quad \forall(x, \tau),\left(x^{\prime}, \tau^{\prime}\right) \in M \times \mathbb{S}^{1}
$$

and

$$
h_{\tau, t}(x, y) \leq h_{\tau, s}(x, z)+\Phi_{s, t}(z, y), \quad \forall(x, \tau),(y, t),(z, s) \in M \times \mathbb{S}^{1} .
$$

It can be shown that the extended Peierls barrier $h_{\tau, \tau^{\prime}}$ is Lipschitz and that, the liminf in (3.1) can not always be replaced with a limit, which leads to the nonconvergence of the L-O semigroup associated with $L$ [18. See [37] for more details about the action potential and the extended Peierls barrier. Before stating Proposition 3.5 we introduce the following lemma. 
Lemma 3.4 (A Priori Compactness). If $t>0$ is fixed, there exists a compact subset $\mathcal{C}_{t} \subset T M \times \mathbb{S}^{1}$ such that for each minimizing extremal curve $\gamma:[a, b] \rightarrow M$ with $b-a \geq t$, we have

$$
(\gamma(s), \dot{\gamma}(s),[s]) \in \mathcal{C}_{t}, \quad \forall s \in[a, b] .
$$

The lemma may be proved by small modifications of the proof found in [19, Corollary 4.3.2].

\section{Proposition 3.5.}

$$
\lim _{n \rightarrow+\infty} \inf _{\substack{k \in \mathbb{N} \\ n \leq k \leq 2 n}} F_{\tau, \tau^{\prime}+k}\left(x, x^{\prime}\right)=h_{\tau, \tau^{\prime}}\left(x, x^{\prime}\right)
$$

uniformly on $\left(\tau, \tau^{\prime}, x, x^{\prime}\right) \in \mathbb{S}^{1} \times \mathbb{S}^{1} \times M \times M$.

Proof. Throughout this proof we use $C$ to denote a generic positive constant not necessarily the same in any two places. Since the proof is rather long, it is convenient to divide it into two steps.

Step 1. In the first step, we show that

$$
\lim _{n \rightarrow+\infty} \inf _{\substack{k \in \mathbb{N} \\ n \leq k \leq 2 n}} F_{\tau, \tau^{\prime}+k}\left(x, x^{\prime}\right)=h_{\tau, \tau^{\prime}}\left(x, x^{\prime}\right), \quad \forall\left(\tau, \tau^{\prime}, x, x^{\prime}\right) \in \mathbb{S}^{1} \times \mathbb{S}^{1} \times M \times M .
$$

For each $\tau, \tau^{\prime} \in \mathbb{S}^{1}$ and each $x, x^{\prime} \in M$, by the definition of $h_{\tau, \tau^{\prime}}$, we have $\liminf _{k \rightarrow+\infty} F_{\tau, \tau^{\prime}+k}\left(x, x^{\prime}\right)=h_{\tau, \tau^{\prime}}\left(x, x^{\prime}\right)$. Then there exist $\left\{k_{i}\right\}_{i=1}^{+\infty}$ such that $k_{i} \rightarrow$ $+\infty$ and $F_{\tau, \tau^{\prime}+k_{i}}\left(x, x^{\prime}\right) \rightarrow h_{\tau, \tau^{\prime}}\left(x, x^{\prime}\right)$ as $i \rightarrow+\infty$. Tonelli's theorem guarantees the existence of the minimizing extremal curves $\gamma_{k_{i}}:\left[\tau, \tau^{\prime}+k_{i}\right] \rightarrow M$ with $\gamma_{k_{i}}(\tau)=x$, $\gamma_{k_{i}}\left(\tau^{\prime}+k_{i}\right)=x^{\prime}$ and $A\left(\gamma_{k_{i}}\right)=F_{\tau, \tau^{\prime}+k_{i}}\left(x, x^{\prime}\right)$, where

$$
A\left(\gamma_{k_{i}}\right)=\int_{\tau}^{\tau^{\prime}+k_{i}} L\left(\gamma_{k_{i}}, \dot{\gamma}_{k_{i}}, s\right) d s
$$

Thus, we have $A\left(\gamma_{k_{i}}\right) \rightarrow h_{\tau, \tau^{\prime}}\left(x, x^{\prime}\right)$ as $i \rightarrow+\infty$. Then for every $\varepsilon>0$, there exists $I \in \mathbb{N}$ such that

$$
\left|A\left(\gamma_{k_{i}}\right)-h_{\tau, \tau^{\prime}}\left(x, x^{\prime}\right)\right|<\varepsilon
$$

if $i \geq I, i \in \mathbb{N}$. And it is clear that for each $k_{i},\left(\gamma_{k_{i}}(s), \dot{\gamma}_{k_{i}}(s),[s]\right):\left[\tau, \tau^{\prime}+k_{i}\right] \rightarrow$ $T M \times \mathbb{S}^{1}$ is a trajectory of the Euler-Lagrange flow.

To prove (3.4), it suffices to show that for $n \in \mathbb{N}$ large enough, we can find a curve $\tilde{\gamma}:\left[\tau, \tau^{\prime}+k_{0}\right] \rightarrow M$ with $\tilde{\gamma}(\tau)=x, \tilde{\gamma}\left(\tau^{\prime}+k_{0}\right)=x^{\prime}$, where $n \leq k_{0} \leq 2 n$, $k_{0} \in \mathbb{N}$, such that

$$
\left|A(\tilde{\gamma})-A\left(\gamma_{k_{I}}\right)\right| \leq C \varepsilon
$$

for some constant $C>0$. In fact, if such a curve exists, then 
$\inf _{\substack{k \in \mathbb{N} \\ n \leq k}} F_{\tau, \tau^{\prime}+k}\left(x, x^{\prime}\right) \leq \inf _{\substack{k \in \mathbb{N} \\ n \leq k \leq 2 n}} F_{\tau, \tau^{\prime}+k}\left(x, x^{\prime}\right) \leq A(\tilde{\gamma}) \leq A\left(\gamma_{k_{I}}\right)+C \varepsilon \leq h_{\tau, \tau^{\prime}}\left(x, x^{\prime}\right)+C \varepsilon$.

By letting $n \rightarrow+\infty$, from the arbitrariness of $\varepsilon>0$, we have

$$
\begin{aligned}
h_{\tau, \tau^{\prime}}\left(x, x^{\prime}\right) & =\liminf _{k \rightarrow+\infty} F_{\tau, \tau^{\prime}+k}\left(x, x^{\prime}\right) \\
& =\lim _{n \rightarrow+\infty} \inf _{\substack{k \in \mathbb{N} \\
n \leq k}} F_{\tau, \tau^{\prime}+k}\left(x, x^{\prime}\right) \\
& \leq \lim _{n \rightarrow+\infty} \inf _{\substack{k \in \mathbb{N} \\
n \leq k \leq 2 n}} F_{\tau, \tau^{\prime}+k}\left(x, x^{\prime}\right) \\
& \leq h_{\tau, \tau^{\prime}}\left(x, x^{\prime}\right),
\end{aligned}
$$

which implies that

$$
\lim _{n \rightarrow+\infty} \inf _{\substack{k \in \mathbb{N} \\ n \leq k \leq 2 n}} F_{\tau, \tau^{\prime}+k}\left(x, x^{\prime}\right)=h_{\tau, \tau^{\prime}}\left(x, x^{\prime}\right) .
$$

Our task is now to construct the curve mentioned above. Note that for the above $\varepsilon>0$, there exists $I^{\prime} \in \mathbb{N}$ such that there exists

$$
\left(z_{k_{i}}, v_{z_{k_{i}}}, t_{z_{k_{i}}}\right) \in O_{i}:=\left\{\left(\gamma_{k_{i}}(s), \dot{\gamma}_{k_{i}}(s),[s]\right) \mid \tau \leq s \leq \tau^{\prime}+k_{i}\right\} \subset T M \times \mathbb{S}^{1}
$$

such that

$$
d\left(\left(z_{k_{i}}, v_{z_{k_{i}}}, t_{z_{k_{i}}}\right), \tilde{\mathcal{M}}_{0}\right)<\varepsilon
$$

if $i \geq I^{\prime}, i \in \mathbb{N}$, where $\tilde{\mathcal{M}}_{0}$ is the Mather set of cohomology class 0. As usual, distance is measured with respect to smooth Riemannian metrics. Since $\tilde{\mathcal{M}}_{0}$ is compact and by the a priori compactness given by Lemma 3.4. $O_{i}$ is contained in the compact subset $\mathcal{C}_{k_{I^{\prime}-1}}$ of $T M \times \mathbb{S}^{1}$ for each $i \geq I^{\prime}$, then it doesn't matter which Riemannian metrics we choose to measure distance.

Let $I=\max \left\{I, I^{\prime}\right\}$. Then $\left|A\left(\gamma_{k_{I}}\right)-h_{\tau, \tau^{\prime}}\left(x, x^{\prime}\right)\right|<\varepsilon$ and there exists $\left(z_{0}, v_{z_{0}}, t_{z_{0}}\right) \in$ $O_{I}=\left\{\left(\gamma_{k_{I}}(s), \dot{\gamma}_{k_{I}}(s),[s]\right) \mid \tau \leq s \leq \tau^{\prime}+k_{I}\right\}$ such that

$$
d\left(\left(z_{0}, v_{z_{0}}, t_{z_{0}}\right), \tilde{\mathcal{M}}_{0}\right)<\varepsilon
$$

In view of (3.5), there exists an ergodic minimal measure $\mu_{e}$ on $T M \times \mathbb{S}^{1}$ 33. such that $\mu_{e}\left(\operatorname{supp} \mu_{e} \cap B_{2 \varepsilon}\left(z_{0}, v_{z_{0}}, t_{z_{0}}\right)\right)=\Delta>0$, where $B_{2 \varepsilon}\left(z_{0}, v_{z_{0}}, t_{z_{0}}\right)$ denotes the open ball of radius $2 \varepsilon$ centered on $\left(z_{0}, v_{z_{0}}, t_{z_{0}}\right)$ in $T M \times \mathbb{S}^{1}$. Set $A_{2 \varepsilon}=\operatorname{supp} \mu_{e} \cap$ $B_{2 \varepsilon}\left(z_{0}, v_{z_{0}}, t_{z_{0}}\right)$. Since $\mu_{e}$ is an ergodic measure, then

$$
\mu_{e}\left(\bigcup_{t=1}^{+\infty} \phi_{-t}^{L}\left(A_{2 \varepsilon}\right)\right)=1
$$


Thus, for any $0<\Delta^{\prime}<\Delta$, there exists $T>0$ such that

$$
\mu_{e}\left(\bigcup_{t=1}^{T^{\prime}} \phi_{-t}^{L}\left(A_{2 \varepsilon}\right)\right) \geq 1-\Delta^{\prime}
$$

if $T^{\prime} \geq T$. From this, we may deduce that for each $n \in \mathbb{N}$,

$$
\left(\bigcup_{t=1}^{T} \phi_{-t}^{L}\left(A_{2 \varepsilon}\right)\right) \cap \phi_{n}^{L}\left(A_{2 \varepsilon}\right) \neq \emptyset .
$$

For, otherwise, there would be $n_{0} \in \mathbb{N}$ such that

$$
\begin{aligned}
\mu_{e}\left(\left(\bigcup_{t=1}^{T} \phi_{-t}^{L}\left(A_{2 \varepsilon}\right)\right) \cup \phi_{n_{0}}^{L}\left(A_{2 \varepsilon}\right)\right) & =\mu_{e}\left(\bigcup_{t=1}^{T} \phi_{-t}^{L}\left(A_{2 \varepsilon}\right)\right)+\mu_{e}\left(\phi_{n_{0}}^{L}\left(A_{2 \varepsilon}\right)\right) \\
& \geq 1-\Delta^{\prime}+\Delta>1,
\end{aligned}
$$

which contradicts that $\mu_{e}$ is a probability measure.

For a given $n \in \mathbb{N}$ large enough with $\max \left\{k_{I}, T+1\right\} \leq\left\{\frac{n}{2}\right\}$, from (3.6) there exist $\left(e_{0}, v_{e_{0}}, t_{e_{0}}\right),\left(\bar{e}_{0}, v_{\bar{e}_{0}}, t_{\bar{e}_{0}}\right) \in A_{2 \varepsilon}$ and $1 \leq t \leq T$ such that

$$
\phi_{-t}^{L}\left(e_{0}, v_{e_{0}}, t_{e_{0}}\right)=\left(e, v_{e}, t_{e}\right)=\phi_{n}^{L}\left(\bar{e}_{0}, v_{\bar{e}_{0}}, t_{\bar{e}_{0}}\right)
$$

for some $\left(e, v_{e}, t_{e}\right) \in \tilde{\mathcal{M}}_{0}$. Since $\left(e_{0}, v_{e_{0}}, t_{e_{0}}\right) \in A_{2 \varepsilon}$, then

$$
d\left(\left(e_{0}, v_{e_{0}}, t_{e_{0}}\right),\left(z_{0}, v_{z_{0}}, t_{z_{0}}\right)\right)<2 \varepsilon
$$

Set $\left(z_{1}, v_{z_{1}}, t_{z_{1}}\right)=\phi_{t_{e_{0}}-t_{z_{0}}}^{L}\left(z_{0}, v_{z_{0}}, t_{z_{0}}\right)$. Then $t_{z_{1}}=t_{e_{0}}$ and from (3.8) we have

$$
d\left(\left(e_{0}, v_{e_{0}}, t_{e_{0}}\right),\left(z_{1}, v_{z_{1}}, t_{e_{0}}\right)\right)<C \varepsilon
$$

for some constant $C>0$. Set $\left(z_{2}, v_{z_{2}}, \tau\right)=\phi_{\tau-t_{e_{0}}}^{L}\left(z_{1}, v_{z_{1}}, t_{e_{0}}\right)$ and $\left(e_{1}, v_{e_{1}}, \tau\right)=$ $\phi_{\tau-t_{e_{0}}}^{L}\left(e_{0}, v_{e_{0}}, t_{e_{0}}\right)$. Then by the differentiability of the solutions of the EulerLagrange equation with respect to initial values, we have

$$
d\left(\left(e_{1}, v_{e_{1}}, \tau\right),\left(z_{2}, v_{z_{2}}, \tau\right)\right)<C \varepsilon
$$

for some constant $C>0$.

Since $\left(e_{0}, v_{e_{0}}, t_{e_{0}}\right),\left(\bar{e}_{0}, v_{\bar{e}_{0}}, t_{\bar{e}_{0}}\right) \in A_{2 \varepsilon}$, then

$$
d\left(\left(e_{0}, v_{e_{0}}, t_{e_{0}}\right),\left(\bar{e}_{0}, v_{\bar{e}_{0}}, t_{\bar{e}_{0}}\right)\right)<4 \varepsilon .
$$

Set $\left(\bar{e}_{1}, v_{\bar{e}_{1}}, t_{e_{0}}\right)=\phi_{t_{e_{0}}-t_{\bar{e}_{0}}}^{L}\left(\bar{e}_{0}, v_{\bar{e}_{0}}, t_{\bar{e}_{0}}\right)$. Then from (3.11) we have 


$$
d\left(\left(e_{0}, v_{e_{0}}, t_{e_{0}}\right),\left(\bar{e}_{1}, v_{\bar{e}_{1}}, t_{e_{0}}\right)\right)<C \varepsilon
$$

for some constant $C>0$. Set $\left(\bar{e}_{2}, v_{\bar{e}_{2}}, \tau\right)=\phi_{\tau-t_{e_{0}}}^{L}\left(\bar{e}_{1}, v_{\bar{e}_{1}}, t_{e_{0}}\right)$. Recall that $\left(e_{1}, v_{e_{1}}, \tau\right)=\phi_{\tau-t_{e_{0}}}^{L}\left(e_{0}, v_{e_{0}}, t_{e_{0}}\right)$. Then from the differentiability of the solutions of the Euler-Lagrange equation with respect to initial values, we have

$$
d\left(\left(e_{1}, v_{e_{1}}, \tau\right),\left(\bar{e}_{2}, v_{\bar{e}_{2}}, \tau\right)<C \varepsilon\right.
$$

for some constant $C>0$.

Note that since $\left(z_{0}, v_{z_{0}}, t_{z_{0}}\right) \in O_{I}=\left\{\left(\gamma_{k_{I}}(s), \dot{\gamma}_{k_{I}}(s),[s]\right) \mid \tau \leq s \leq \tau^{\prime}+k_{I}\right\}$, where $O_{I}$ is an orbit of the Euler-Lagrange flow, then $\left(z_{2}, v_{z_{2}}, \tau\right) \in O_{I}$. And thus, there exists $k_{I_{1}}, k_{I_{2}} \in \mathbb{N}$ with $k_{I_{1}}+k_{I_{2}}=k_{I}$ such that

$$
\left(z_{2}, v_{z_{2}}, \tau\right)=\left(\gamma_{k_{I}}\left(\tau+k_{I_{1}}\right), \dot{\gamma}_{k_{I}}\left(\tau+k_{I_{1}}\right), \tau\right) .
$$

We are now in a position to construct the curve we need. We treat the case $k_{I_{1}} \neq 0, k_{I_{2}} \neq 0$ and the remaining cases can be treated similarly. Let $\alpha_{1}:[\tau, \tau+$ $\left.k_{I_{1}}\right] \rightarrow M$ with $\alpha_{1}(\tau)=x$ and $\alpha_{1}\left(\tau+k_{I_{1}}\right)=\bar{e}_{2}$ be a Tonelli minimizer such that $A\left(\alpha_{1}\right)=F_{\tau, \tau+k_{I_{1}}}\left(x, \bar{e}_{2}\right)$. Since $\gamma_{k_{I}}:\left[\tau, \tau^{\prime}+k_{I}\right] \rightarrow M$ is a minimizing extremal curve, then $\left.\gamma_{k_{I}}\right|_{\left[\tau, \tau+k_{I_{1}}\right]}$ is also a minimizing extremal curve and thus $A\left(\left.\gamma_{k_{I}}\right|_{\left[\tau, \tau+k_{I_{1}}\right]}\right)=$ $F_{\tau, \tau+k_{I_{1}}}\left(x, z_{2}\right)$. Therefore, by the Lipschtiz property of the function $F_{\tau, \tau+k_{I_{1}}}$ (see, for example, [3]), (3.10) and (3.13) we have

$$
\left|A\left(\alpha_{1}\right)-A\left(\left.\gamma_{k_{I}}\right|_{\left[\tau, \tau+k_{I_{1}}\right]}\right)\right|=\left|F_{\tau, \tau+k_{I_{1}}}\left(x, \bar{e}_{2}\right)-F_{\tau, \tau+k_{I_{1}}}\left(x, z_{2}\right)\right| \leq \operatorname{Dd}\left(\bar{e}_{2}, z_{2}\right) \leq C \varepsilon
$$

for some constant $C>0$, where $D>0$ is a Lipschitz constant of $F_{t_{1}, t_{2}}$ which is independent of $t_{1}, t_{2}$ with $t_{1}+1 \leq t_{2}$.

Let $\beta(s)=p \phi_{s-\left(\tau+k_{I_{1}}\right)}^{L}\left(\bar{e}_{2}, v_{\bar{e}_{2}}, \tau\right), s \in \mathbb{R}^{1}$, where $p: T M \times \mathbb{S}^{1} \rightarrow M$ denotes the projection. Then $(\beta(s), \dot{\beta}(s),[s])=\phi_{s-\left(\tau+k_{I_{1}}\right)}^{L}\left(\bar{e}_{2}, v_{\bar{e}_{2}}, \tau\right), s \in \mathbb{R}^{1}$, and $(\beta(\tau+$ $\left.\left.k_{I_{1}}\right), \dot{\beta}\left(\tau+k_{I_{1}}\right)\right)=\left(\bar{e}_{2}, v_{\bar{e}_{2}}\right)$. Hence, from (3.7) we have

$$
\left(e, v_{e}, t_{e}\right)=(\beta(l), \dot{\beta}(l),[l]),
$$

where $l=\tau+k_{I_{1}}+\left(t_{e_{0}}-\tau\right)+\left(t_{\bar{e}_{0}}-t_{e_{0}}\right)+n$, and

$$
\left(e_{1}, v_{e_{1}}, \tau\right)=\left(\beta\left(l^{\prime}\right), \dot{\beta}\left(l^{\prime}\right),\left[l^{\prime}\right]\right),
$$

where $l^{\prime}=l+t+\left(\tau-t_{e_{0}}\right)=\tau+k_{I_{1}}+n+t+t_{\bar{e}_{0}}-t_{e_{0}}$. Then $\left[l^{\prime}\right]=\left[\tau+k_{I_{1}}+n+\right.$ $\left.t+t_{\bar{e}_{0}}-t_{e_{0}}\right]=\left[\tau+t+t_{\bar{e}_{0}}-t_{e_{0}}\right]=\tau$, which means that $t+t_{\bar{e}_{0}}-t_{e_{0}} \in \mathbb{Z}$. Notice that $0 \leq t+t_{\bar{e}_{0}}-t_{e_{0}} \leq T+t_{\bar{e}_{0}}-t_{e_{0}} \leq\left\{\frac{n}{2}\right\}$. Thus, 


$$
n \leq k_{I}+n+t+t_{\bar{e}_{0}}-t_{e_{0}} \leq k_{I}+n+\left\{\frac{n}{2}\right\} \leq 2 n .
$$

Let $m=n+t+t_{\bar{e}_{0}}-t_{e_{0}} \in \mathbb{Z}$ and $\alpha_{2}=\left.\beta\right|_{\left[\tau+k_{I_{1}}, \tau+k_{I_{1}}+m\right]}$. Then $\alpha_{2}\left(\tau+k_{I_{1}}\right)=$ $\beta\left(\tau+k_{I_{1}}\right)=\bar{e}_{2}$ and $\alpha_{2}\left(\tau+k_{I_{1}}+m\right)=\beta\left(\tau+k_{I_{1}}+m\right)=e_{1}$. In view of $\left(\bar{e}_{0}, v_{\bar{e}_{0}}, t_{\bar{e}_{0}}\right) \in$ $A_{2 \varepsilon} \subset \tilde{\mathcal{M}}_{0}$ and the definitions of $\beta$ and $\alpha_{2},\left(\alpha_{2}(s), \dot{\alpha}_{2}(s),[s]\right)$ is a trajectory of the Euler-Lagrange flow in $\tilde{\mathcal{M}}_{0}$. According to [33, Proposition 3] and the definition of $h_{\tau, \tau}$, we have

$$
A\left(\alpha_{2}\right)=F_{\tau+k_{I_{1}}, \tau+k_{I_{1}}+m}\left(\bar{e}_{2}, e_{1}\right)=h_{\tau, \tau}\left(\bar{e}_{2}, e_{1}\right) .
$$

Hence, on account of the Lipschitz property of $h_{\tau, \tau}$ and (3.13),

$$
\left|A\left(\alpha_{2}\right)-h_{\tau, \tau}\left(e_{1}, e_{1}\right)\right|=\left|h_{\tau, \tau}\left(\bar{e}_{2}, e_{1}\right)-h_{\tau, \tau}\left(e_{1}, e_{1}\right)\right| \leq \bar{D} d\left(\bar{e}_{2}, e_{1}\right) \leq C \varepsilon
$$

for some constant $C>0$, where $\bar{D}$ is a Lipschitz constant of $h_{\tau, \tau}$. Since $\left(e_{1}, \tau\right) \in$ $\mathcal{M}_{0}$, where $\mathcal{M}_{0} \subset M \times \mathbb{S}^{1}$ is the projected Mather set, then $h_{\tau, \tau}\left(e_{1}, e_{1}\right)=0$, and thus

$$
\left|A\left(\alpha_{2}\right)\right| \leq C \varepsilon
$$

Let $\alpha_{3}:\left[\tau+k_{I_{1}}+m, \tau^{\prime}+k_{I}+m\right] \rightarrow M$ with $\alpha_{3}\left(\tau+k_{I_{1}}+m\right)=e_{1}$ and $\alpha_{3}\left(\tau^{\prime}+k_{I}+m\right)=x^{\prime}$ be a Tonelli minimizer such that

$$
A\left(\alpha_{3}\right)=F_{\tau+k_{I_{1}}+m, \tau^{\prime}+k_{I}+m}\left(e_{1}, x^{\prime}\right)=F_{\tau+k_{I_{1}}, \tau^{\prime}+k_{I}}\left(e_{1}, x^{\prime}\right) .
$$

Since $\gamma_{k_{I}}:\left[\tau, \tau^{\prime}+k_{I}\right] \rightarrow M$ is a minimizing extremal curve, then $\left.\gamma_{k_{I}}\right|_{\left[\tau+k_{I_{1}}, \tau^{\prime}+k_{I}\right]}$ is also a minimizing extremal curve and thus

$$
A\left(\left.\gamma_{k_{I}}\right|_{\left[\tau+k_{I_{1}}, \tau^{\prime}+k_{I}\right]}\right)=F_{\tau+k_{I_{1}}, \tau^{\prime}+k_{I}}\left(z_{2}, x^{\prime}\right) .
$$

Therefore, from the Lipschitz property of $F_{\tau+k_{I_{1}}, \tau^{\prime}+k_{I}}$ and (3.10), we have

$$
\begin{aligned}
\left|A\left(\alpha_{3}\right)-A\left(\left.\gamma_{k_{I}}\right|_{\left[\tau+k_{I_{1}}, \tau^{\prime}+k_{I}\right]}\right)\right| & =\left|F_{\tau+k_{I_{1}}, \tau^{\prime}+k_{I}}\left(e_{1}, x^{\prime}\right)-F_{\tau+k_{I_{1}}, \tau^{\prime}+k_{I}}\left(z_{2}, x^{\prime}\right)\right| \\
& \leq \operatorname{Dd}\left(e_{1}, z_{2}\right) \\
& \leq C \varepsilon
\end{aligned}
$$

for some constant $C>0$.

Consider the curve $\tilde{\gamma}:\left[\tau, \tau^{\prime}+k_{I}+m\right] \rightarrow M$ connecting $x$ and $x^{\prime}$ defined by 


$$
\tilde{\gamma}(s)= \begin{cases}\alpha_{1}(s), & s \in\left[\tau, \tau+k_{I_{1}}\right], \\ \alpha_{2}(s), & s \in\left[\tau+k_{I_{1}}, \tau+k_{I_{1}}+m\right], \\ \alpha_{3}(s), & s \in\left[\tau+k_{I_{1}}+m, \tau^{\prime}+k_{I}+m\right] .\end{cases}
$$

By (3.15), $n \leq k_{0}:=k_{I}+m \leq 2 n$. From (3.14), (3.16) and (3.17), we have

$$
\left|A(\tilde{\gamma})-A\left(\gamma_{k_{I}}\right)\right| \leq C \varepsilon
$$

for some constant $C>0$. It is clear that $\tilde{\gamma}$ is just the curve we need, and we have proved (3.4).

Step 2. For each $n \in \mathbb{N}$ and each $\left(\tau, \tau^{\prime}, x, x^{\prime}\right) \in[0,1] \times[0,1] \times M \times M$, let

$$
\mathcal{F}_{n}\left(\tau, \tau^{\prime}, x, x^{\prime}\right)=\inf _{\substack{k \in \mathbb{N} \\ n \leq k \leq 2 n}} F_{\tau, \tau^{\prime}+k}\left(x, x^{\prime}\right) .
$$

Then, to complete the proof of Proposition 3.5 it suffices to show that $\left\{\mathcal{F}_{n}\right\}_{n=2}^{+\infty}$ are equicontinuous. Notice that $\left(\tau, \tau^{\prime}, x, x^{\prime}\right) \mapsto F_{\tau, \tau^{\prime}+k}\left(x, x^{\prime}\right)$ is a Lipschitz function on $[0,1] \times[0,1] \times M \times M$ for every $k \geq 2, k \in \mathbb{N}$, and that the Lipschitz constant $\tilde{D}$ is independent of $k$, see [3, 3.3 LEMMA]. Hence, for each $n \geq 2, n \in \mathbb{N}$ the function $\left(\tau, \tau^{\prime}, x, x^{\prime}\right) \mapsto \mathcal{F}_{n}\left(\tau, \tau^{\prime}, x, x^{\prime}\right)$ is also Lipschitz with the same Lipschitz constant $\tilde{D}$, and thus $\left\{\mathcal{F}_{n}\right\}_{n=2}^{+\infty}$ are equicontinuous. The proof is now complete.

Recall that for each $n \in \mathbb{N}$ and each $u \in C\left(M, \mathbb{R}^{1}\right)$,

$U_{n}^{u}(x, \tau)=\tilde{T}_{n}^{\tau} u(x)=\inf _{\substack{k \in \mathbb{N} \\ n \leq k \leq 2 n}} \inf _{y \in M}\left(u(y)+F_{0, \tau+k}(y, x)\right)=\inf _{y \in M}\left(u(y)+\mathcal{F}_{n}(0, \tau, y, x)\right)$

for all $(x, \tau) \in M \times[0,1]$. Since

$$
\begin{aligned}
\left|U_{n}^{u}(x, \tau)-\inf _{y \in M}\left(u(y)+h_{0, \tau}(y, x)\right)\right| & =\left|\inf _{y \in M}\left(u(y)+\mathcal{F}_{n}(0, \tau, y, x)\right)-\inf _{y \in M}\left(u(y)+h_{0, \tau}(y, x)\right)\right| \\
& \leq \sup _{y \in M}\left|\mathcal{F}_{n}(0, \tau, y, x)-h_{0, \tau}(y, x)\right|,
\end{aligned}
$$

then from Proposition 3.5. we conclude that the uniform limit $\bar{u}=\lim _{n \rightarrow+\infty} U_{n}^{u}$ exists, and

$$
\bar{u}(x, \tau)=\inf _{y \in M}\left(u(y)+h_{0, \tau}(y, x)\right)
$$

for all $(x, \tau) \in M \times \mathbb{S}^{1}$, thus proving the first assertion of Theorem 1.2. 
K. WANG AND J. YAN

3.3. $\lim _{n \rightarrow+\infty} U_{n}^{u}$, backward weak KAM solutions and viscosity solutions. Here we discuss the relation among uniform limits $\lim _{n \rightarrow+\infty} U_{n}^{u}$, backward weak KAM solutions and viscosity solutions of (1.5). Following Fathi [14], as done by Contreras et al. in [9, we give the definition of the backward weak KAM solution as follows.

Definition 3.6. A backward weak KAM solution of the Hamilton-Jacobi equation (1.5) is a function $u: M \times \mathbb{S}^{1} \rightarrow \mathbb{R}^{1}$ such that

(1) $\mathrm{u}$ is dominated by $L$, i.e.,

$$
u(x, \tau)-u(y, s) \leq \Phi_{s, \tau}(y, x), \quad \forall(x, \tau),(y, s) \in M \times \mathbb{S}^{1} .
$$

We use the notation $u \prec L$.

(2) For every $(x, \tau) \in M \times \mathbb{S}^{1}$ there exists a curve $\gamma:(-\infty, \tilde{\tau}] \rightarrow M$ with $\gamma(\tilde{\tau})=x$ and $[\tilde{\tau}]=\tau$ such that

$$
u(x, \tau)-u(\gamma(t),[t])=\int_{t}^{\tilde{\tau}} L(\gamma(s), \dot{\gamma}(s), s) d s, \quad \forall t \in(-\infty, \tilde{\tau}] .
$$

We denote by $\mathcal{S}_{-}$the set of backward weak KAM solutions. Let us recall two known results [9] on backward weak KAM solutions, which will be used later in the paper.

Lemma 3.7. Given a fixed $(y, s) \in M \times \mathbb{S}^{1}$, the function

$$
(x, \tau) \mapsto h_{s, \tau}(y, x), \quad(x, \tau) \in M \times \mathbb{S}^{1}
$$

is a backward weak KAM solution.

Lemma 3.8. If $\mathcal{U} \subset \mathcal{S}_{-}$, let $\underline{u}(x, \tau):=\inf _{u \in \mathcal{U}} u(x, \tau)$ then either $\underline{u} \equiv-\infty$ or $\underline{u} \in \mathcal{S}_{-}$.

We define the projected Aubry set $\mathcal{A}_{0}$ as follows:

$$
\mathcal{A}_{0}:=\left\{(x, \tau) \in M \times \mathbb{S}^{1} \mid h_{\tau, \tau}(x, x)=0\right\} .
$$

Note that $\mathcal{A}_{0}=\Pi \tilde{\mathcal{A}}_{0}$, where $\Pi: T M \times \mathbb{S}^{1} \rightarrow M \times \mathbb{S}^{1}$ denotes the projection and $\tilde{\mathcal{A}}_{0}$ denotes the Aubry set in $T M \times \mathbb{S}^{1}$, i.e., the union of global static orbits. See for instance [3] for the definition of static orbits and more details on $\tilde{\mathcal{A}}_{0}$.

From the definition of $\mathcal{A}_{0},(3.2)$ and (3.3), it is straightforward to show that if $(x, \tau) \in \mathcal{A}_{0}$, then

$$
h_{\tau, s}(x, y)=\Phi_{\tau, s}(x, y)
$$

for all $(y, s) \in M \times \mathbb{S}^{1}$. Define an equivalence relation on $\mathcal{A}_{0}$ by saying that $(x, \tau)$ and $(y, s)$ are equivalent if and only if

$$
\Phi_{\tau, s}(x, y)+\Phi_{s, \tau}(y, x)=0
$$


By (3.19), it is simple to see that (3.20) is equivalent to

$$
h_{\tau, s}(x, y)+h_{s, \tau}(y, x)=0 .
$$

The equivalent classes of this relation are called static classes. Let $\mathrm{A}$ be the set of static classes. For each static class $\Gamma \in \mathrm{A}$ choose a point $(x, 0) \in \Gamma$ and let $\mathbb{A}_{0}$ be the set of such points.

Contreras et al. 9] characterize backward weak KAM solutions of the HamiltonJacobi equation (1.5) in terms of their values at each static class and the extended Peierls barrier. See [8] for similar results in the time-independent case.

Theorem 3.9 (Contreras et al. 9]). The $\operatorname{map}\left\{f: \mathbb{A}_{0} \rightarrow \mathbb{R}^{1} \mid f \prec L\right\} \rightarrow \mathcal{S}_{-}$

$$
f \mapsto u_{f}(x, \tau)=\min _{(p, 0) \in \mathbb{A}_{0}}\left(f(p, 0)+h_{0, \tau}(p, x)\right)
$$

is a bijection.

\section{Proposition 3.10.}

$$
\left\{\bar{u} \in C\left(M \times \mathbb{S}^{1}, \mathbb{R}^{1}\right) \mid \exists u \in C\left(M, \mathbb{R}^{1}\right), \bar{u}=\lim _{n \rightarrow+\infty} U_{n}^{u}\right\}=\mathcal{S}_{-} .
$$

Remark 3.11. Proposition 3.10 tells us two things: (i) For each $u \in C\left(M, \mathbb{R}^{1}\right)$, $\bar{u}=\lim _{n \rightarrow+\infty} U_{n}^{u}$ is a backward weak KAM solution of (1.5), which proves the second assertion of Theorem 1.2 . (ii) For each $w \in \mathcal{S}_{-}$there exists $w_{0} \in C\left(M, \mathbb{R}^{1}\right)$ such that $w=\lim _{n \rightarrow+\infty} U_{n}^{w_{0}}$. Moreover, we know from the proof of Proposition 3.10 that $w_{0}(x)=w(x, 0)$ for all $x \in M$.

Proof. First we show that for each $u \in C\left(M, \mathbb{R}^{1}\right), \bar{u}=\lim _{n \rightarrow+\infty} U_{n}^{u}$ is a backward weak KAM solution of (1.5). By (3.18) we have

$$
\bar{u}(x, \tau)=\inf _{y \in M}\left(u(y)+h_{0, \tau}(y, x)\right)
$$

for all $(x, \tau) \in M \times \mathbb{S}^{1}$. Combining Lemmas 3.7 and 3.8 we get that $\bar{u} \in \mathcal{S}_{-}$.

Then we prove that for each $w \in \mathcal{S}_{-}$, there exists $w_{0} \in C\left(M, \mathbb{R}^{1}\right)$ such that $w=\lim _{n \rightarrow+\infty} U_{n}^{w_{0}}$. From Theorem 3.9 there exists $f: \mathbb{A}_{0} \rightarrow \mathbb{R}^{1}$ with $f \prec L$ such that for each $(x, \tau) \in M \times \mathbb{S}^{1}$,

$$
\begin{aligned}
w(x, \tau) & =\min _{(p, 0) \in \mathbb{A}_{0}}\left(f(p, 0)+h_{0, \tau}(p, x)\right) \\
& =\min _{(p, 0) \in \mathbb{A}_{0}}\left(f(p, 0)+\min _{y \in M}\left(h_{0,0}(p, y)+h_{0, \tau}(y, x)\right)\right) \\
& =\min _{y \in M}\left(\min _{(p, 0) \in \mathbb{A}_{0}}\left(f(p, 0)+h_{0,0}(p, y)\right)+h_{0, \tau}(y, x)\right) \\
& =\min _{y \in M}\left(w(y, 0)+h_{0, \tau}(y, x)\right) .
\end{aligned}
$$


Let $w_{0}(x)=w(x, 0)$ for all $x \in M$. Then by Proposition 3.5 and (3.18), the uniform limit $\bar{w}_{0}=\lim _{n \rightarrow+\infty} U_{n}^{w_{0}}$ exists and

$$
\bar{w}_{0}(x, \tau)=\min _{y \in M}\left(w_{0}(y)+h_{0, \tau}(y, x)\right)=\min _{y \in M}\left(w(y, 0)+h_{0, \tau}(y, x)\right)
$$

for all $(x, \tau) \in M \times \mathbb{S}^{1}$. Therefore, $w=\bar{w}_{0}=\lim _{n \rightarrow+\infty} U_{n}^{w_{0}}$.

Proposition 3.12. Let $u \in C\left(M \times \mathbb{S}^{1}, \mathbb{R}^{1}\right)$. Then $u$ is a backward weak KAM solution of (1.5) if and only if it is a viscosity solution of (1.5).

Proof. Let $u \in C\left(M \times \mathbb{S}^{1}, \mathbb{R}^{1}\right)$ and $u_{0}(x)=u(x, 0)$ for all $x \in M$. If $u$ is a backward weak KAM solution of (1.5), then from Proposition 3.10 we have $u=\lim _{n \rightarrow+\infty} U_{n}^{u_{0}}$. Recall that

$$
U_{n}^{u_{0}}(x, \tau)=\tilde{T}_{n}^{\tau} u_{0}(x)=\left(T_{\tau} \circ \tilde{T}_{n} u_{0}\right)(x) .
$$

It is a standard result that for each $n \in \mathbb{N}, U_{n}^{u_{0}}(x, \tau)=\left(T_{\tau} \circ \tilde{T}_{n} u_{0}\right)(x)$ is a viscosity solution of (1.5), see [18] for instance. Since $u$ is the uniform limit of $\left\{U_{n}^{u_{0}}\right\}_{n=1}^{+\infty}$, then from the stability of viscosity solution of (1.5) [19], $u$ is also a viscosity solution of (1.5).

Suppose now that $u$ is a viscosity solution of (1.5). Let $U^{u_{0}}(x, t)=T_{t} u_{0}(x)$ for all $(x, t) \in M \times[0,+\infty)$. Then $U^{u_{0}}$ is a viscosity solution of (1.5) with $U^{u_{0}}(x, 0)=$ $T_{0} u_{0}(x)=u_{0}(x)$. Since $u$ can be considered as a 1-periodic in time viscosity solution on $M \times[0,+\infty)$ and the Cauchy Problem

$$
\begin{cases}v_{t}+H\left(x, v_{x}, t\right)=0, & \text { on } M \times(0,+\infty), \\ v(x, 0)=u_{0}(x), & \text { on } M\end{cases}
$$

is well posed in the viscosity sense (see, for example, [28] or [4]), then $u(x, t)=$ $U^{u_{0}}(x, t)=T_{t} u_{0}(x)$ for all $(x, t) \in M \times[0,+\infty)$. Since $u$ is 1 -periodic in time, for each $(x, \tau) \in M \times[0,1]$ we have

$$
u(x, \tau)=u(x, \tau+k)=\inf _{\gamma}\left\{u_{0}(\gamma(0))+\int_{0}^{\tau+k} L(\gamma, \dot{\gamma}, s) d s\right\}, \quad \forall k \in \mathbb{N},
$$

where the infimum is taken among the continuous and piecewise $C^{1}$ paths $\gamma$ : $[0, \tau+k] \rightarrow M$ with $\gamma(\tau+k)=x$. Hence,

$$
u(x, \tau)=\inf _{\substack{k \in \mathbb{N} \\ n \leq k \leq 2 n}} \inf _{y \in M}\left(u_{0}(y)+F_{0, \tau+k}(y, x)\right)=U_{n}^{u_{0}}(x, \tau), \quad \forall n \in \mathbb{N} .
$$

Then by letting $n \rightarrow+\infty$, from Proposition 3.10 we have $u=\lim _{n \rightarrow+\infty} U_{n}^{u_{0}} \in \mathcal{S}_{-}$. 


\section{THE NEW L-O OPERATOR: TIME-INDEPENDENT CASE}

As mentioned in the Introduction, in this section we first discuss the main properties of the new L-O semigroup associated with $L_{a}$ and then give the proofs of Theorems 1.5 and 1.6. Finally, we construct an example to show that the new L-O semigroup converges faster than the L-O semigroup in the sense of order when the Aubry set $\tilde{\mathcal{A}}_{0}$ of the Lagrangian system (1.6) is a quasi-periodic invariant torus with Diophantine frequency vector $\omega \in \mathcal{D}(\rho, \alpha)$.

4.1. Main properties of the new L-O semigroup. Let us recall the definition of the new L-O operator $\tilde{T}_{t}^{a}$ associated with $L_{a}$. For each $t \geq 0$ and each $u \in$ $C\left(M, \mathbb{R}^{1}\right)$

$$
\tilde{T}_{t}^{a} u(x)=\inf _{t \leq \sigma \leq 2 t} \inf _{\gamma}\left\{u(\gamma(0))+\int_{0}^{\sigma} L_{a}(\gamma(s), \dot{\gamma}(s)) d s\right\}
$$

for all $x \in M$, where the second infimum is taken among the continuous and piecewise $C^{1}$ paths $\gamma:[0, \sigma] \rightarrow M$ with $\gamma(\sigma)=x$.

Obviously, $\tilde{T}_{t}^{a} u(x)=\inf _{t \leq \sigma \leq 2 t} T_{\sigma}^{a} u(x)$. Moreover, it is straightforward to check that for each $t \geq 0, \tilde{T}_{t}^{a}$ is an operator from $C\left(M, \mathbb{R}^{1}\right)$ to itself, and that $\left\{\tilde{T}_{t}^{a}\right\}_{t \geq 0}$ is a semigroup of operators.

Proposition 4.1. For given $t>0, u \in C\left(M, \mathbb{R}^{1}\right)$ and $x \in M$, there exist $\sigma \in[t, 2 t]$ and a minimizing extremal curve $\gamma:[0, \sigma] \rightarrow M$ such that $\gamma(\sigma)=x$ and

$$
\tilde{T}_{t}^{a} u(x)=u(\gamma(0))+\int_{0}^{\sigma} L_{a}(\gamma, \dot{\gamma}) d s .
$$

Proof. Since $\sigma \mapsto T_{\sigma}^{a} u(x)$ is continuous on $[t, 2 t]$ and $\tilde{T}_{t}^{a} u(x)=\inf _{t \leq \sigma \leq 2 t} T_{\sigma}^{a} u(x)$, then there is $\sigma_{0} \in[t, 2 t]$ such that $\tilde{T}_{t}^{a} u(x)=T_{\sigma_{0}}^{a} u(x)$. From the property of the operator $T_{\sigma_{0}}^{a}$ (see [19, Lemma 4.4.1]), there exists a minimizing extremal curve $\gamma:\left[0, \sigma_{0}\right] \rightarrow M$ such that $\gamma\left(\sigma_{0}\right)=x$ and

$$
\tilde{T}_{t}^{a} u(x)=T_{\sigma_{0}}^{a} u(x)=u(\gamma(0))+\int_{0}^{\sigma_{0}} L_{a}(\gamma, \dot{\gamma}) d s .
$$

Some fundamental properties of $\tilde{T}_{t}^{a}$ are discussed in the following proposition.

\section{Proposition 4.2.}

(1) For $u, v \in C\left(M, \mathbb{R}^{1}\right)$, if $u \leq v$, then $\tilde{T}_{t}^{a} u \leq \tilde{T}_{t}^{a} v, \forall t \geq 0$.

(2) If $c$ is a constant and $u \in C\left(M, \mathbb{R}^{1}\right)$, then $\tilde{T}_{t}^{a}(u+c)=\tilde{T}_{t}^{a} u+c, \forall t \geq 0$.

(3) For each $u, v \in C\left(M, \mathbb{R}^{1}\right)$ and each $t \geq 0,\left\|\tilde{T}_{t}^{a} u-\tilde{T}_{t}^{a} v\right\|_{\infty} \leq\|u-v\|_{\infty}$.

(4) For each $u \in C\left(M, \mathbb{R}^{1}\right), \lim _{t \rightarrow 0^{+}} \tilde{T}_{t}^{a} u=u$.

(5) For each $u \in C\left(M, \mathbb{R}^{1}\right),(t, x) \mapsto \tilde{T}_{t}^{a} u(x)$ is continuous on $[0,+\infty) \times M$.

Remark 4.3. The property (3) means that the semigroup $\left\{\tilde{T}_{t}^{a}\right\}_{t \geq 0}$ is continuous at the origin or of class $C_{0}[25]$. 
Proof. Since $T_{t}^{a}$ has the monotonicity property (see [19, Corollary 4.4.4]), then

$$
\tilde{T}_{t}^{a} u(x)=\inf _{t \leq \sigma \leq 2 t} T_{\sigma}^{a} u(x) \leq \inf _{t \leq \sigma \leq 2 t} T_{\sigma}^{a} v(x)=\tilde{T}_{t}^{a} v(x), \quad \forall t>0, \forall x \in M,
$$

i.e., (1) holds. (2) results from the definition of $\tilde{T}_{t}^{a}$ directly. Note that for any $x \in M$,

$$
-\|u-v\|_{\infty}+v(x) \leq u(x) \leq\|u-v\|_{\infty}+v(x) .
$$

By the properties of $T_{\sigma}^{a}$ (see [19, Corollary 4.4.4]), for each $t \geq 0$ we have

$$
T_{\sigma}^{a} v(x)-\|u-v\|_{\infty} \leq T_{\sigma}^{a} u(x) \leq T_{\sigma}^{a} v(x)+\|u-v\|_{\infty}, \quad \forall \sigma \in[t, 2 t] .
$$

Taking the infimum on $\sigma$ over $[t, 2 t]$ yields

$\inf _{t \leq \sigma \leq 2 t} T_{\sigma}^{a} v(x)-\|u-v\|_{\infty} \leq \inf _{t \leq \sigma \leq 2 t} T_{\sigma}^{a} u(x) \leq \inf _{t \leq \sigma \leq 2 t} T_{\sigma}^{a} v(x)+\|u-v\|_{\infty}, \quad \forall x \in M$, and thus (3) holds.

Next we prove (4). For each $u \in C\left(M, \mathbb{R}^{1}\right)$ and each $\varepsilon>0$, there is $w \in$ $C^{1}\left(M, \mathbb{R}^{1}\right)$ such that $\|u-w\|_{\infty}<\varepsilon$ since $C^{1}\left(M, \mathbb{R}^{1}\right)$ is a dense subset of $C\left(M, \mathbb{R}^{1}\right)$ in the topology of uniform convergence. Thus, we have

$$
\begin{aligned}
\left\|\tilde{T}_{t}^{a} u-u\right\|_{\infty} & \leq\left\|\tilde{T}_{t}^{a} u-\tilde{T}_{t}^{a} w\right\|_{\infty}+\left\|\tilde{T}_{t}^{a} w-w\right\|_{\infty}+\|w-u\|_{\infty} \\
& \leq 2\|w-u\|_{\infty}+\left\|\tilde{T}_{t}^{a} w-w\right\|_{\infty} \\
& \leq 2 \varepsilon+\left\|\tilde{T}_{t}^{a} w-w\right\|_{\infty}, \quad \forall t \geq 0,
\end{aligned}
$$

where we have used (3). Since $M$ is compact, then $w$ is Lipschitz. Denote the Lipschitz constant of $w$ by $K_{w}$, and by the superlinearity of $L_{a}$ there exists $C_{K_{w}} \in$ $\mathbb{R}^{1}$ such that

$$
L_{a}(x, v) \geq K_{w}\|v\|_{x}+C_{K_{w}}, \quad \forall(x, v) \in T M .
$$

For each $x \in M$, each $t \geq 0$ and each continuous and piecewise $C^{1}$ path $\gamma$ : $[0, \sigma] \rightarrow M$ with $\gamma(\sigma)=x$ and $t \leq \sigma \leq 2 t$, since

then

$$
d(\gamma(0), \gamma(\sigma)) \leq \int_{0}^{\sigma}\|\dot{\gamma}(s)\|_{\gamma(s)} d s
$$

$$
\int_{0}^{\sigma} L_{a}(\gamma, \dot{\gamma}) d s \geq K_{w} d(\gamma(0), \gamma(\sigma))+C_{K_{w}} \sigma \geq w(\gamma(\sigma))-w(\gamma(0))+C_{K_{w}} \sigma .
$$

Thus, by the definition of $T_{\sigma}^{a}$ we have

$$
T_{\sigma}^{a} w(x) \geq w(x)+C_{K_{w}} \sigma
$$


Taking the infimum on $\sigma$ over $[t, 2 t]$ on both sides of this last inequality yields

$$
\tilde{T}_{t}^{a} w(x) \geq w(x)+O(t), \quad \text { as } t \rightarrow 0^{+},
$$

where $O(t)$ is independent of $x$. Using the constant curve $\gamma_{x}:[0, \sigma] \rightarrow M, s \mapsto x$, we have

$$
T_{\sigma}^{a} w(x) \leq w(x)+L_{a}(x, 0) \sigma .
$$

Taking the infimum on $\sigma$ over $[t, 2 t]$, we obtain

$$
\tilde{T}_{t}^{a} w(x) \leq w(x)+O(t), \quad \text { as } t \rightarrow 0^{+},
$$

where $O(t)$ is independent of $x$. Combining (4.1), (4.2) and (4.3), we have

$$
\lim _{t \rightarrow 0^{+}}\left\|\tilde{T}_{t}^{a} u-u\right\|_{\infty}=0
$$

i.e., (4) holds.

Finally, we prove (5). For any $\left(t_{0}, x_{0}\right) \in[0,+\infty) \times M$, from the semigroup property and (3) we have

$$
\begin{aligned}
\left|\tilde{T}_{t}^{a} u(x)-\tilde{T}_{t_{0}}^{a} u\left(x_{0}\right)\right| & \leq\left|\tilde{T}_{t}^{a} u(x)-\tilde{T}_{t}^{a} u\left(x_{0}\right)\right|+\left|\tilde{T}_{t}^{a} u\left(x_{0}\right)-\tilde{T}_{t_{0}}^{a} u\left(x_{0}\right)\right| \\
& \leq\left|\tilde{T}_{t}^{a} u(x)-\tilde{T}_{t}^{a} u\left(x_{0}\right)\right|+\left\|\tilde{T}_{t}^{a} u-\tilde{T}_{t_{0}}^{a} u\right\|_{\infty} \\
& \leq\left|\tilde{T}_{t}^{a} u(x)-\tilde{T}_{t}^{a} u\left(x_{0}\right)\right|+\left\|\tilde{T}_{\left|t-t_{0}\right|}^{a} u-u\right\|_{\infty} .
\end{aligned}
$$

From (4.4), $\tilde{T}_{t}^{a} u \in C\left(M, \mathbb{R}^{1}\right)$ and (4), we conclude that (5) holds.

The proposition below establishs a relationship between $\tilde{T}_{t}^{a}$ and $T_{t}^{a}$.

\section{Proposition 4.4 .}

(1) For each $u \in C\left(M, \mathbb{R}^{1}\right)$, the uniform limit $\lim _{t \rightarrow+\infty} \tilde{T}_{t}^{a} u$ exists and

$$
\lim _{t \rightarrow+\infty} \tilde{T}_{t}^{a} u=\lim _{t \rightarrow+\infty} T_{t}^{a} u=\bar{u} .
$$

(2) For each $t \geq 0$ and each $u \in C\left(M, \mathbb{R}^{1}\right),\left\|\tilde{T}_{t}^{a} u-\bar{u}\right\|_{\infty} \leq\left\|T_{t}^{a} u-\bar{u}\right\|_{\infty}$.

(3) $u \in C\left(M, \mathbb{R}^{1}\right)$ is a fixed point of $\left\{\tilde{T}_{t}^{a}\right\}_{t \geq 0}$ if and only if it is a fixed point of $\left\{T_{t}^{a}\right\}_{t \geq 0}$.

Remark 4.5. From (1) $\lim _{t \rightarrow+\infty} \tilde{T}_{t}^{a} u$ exists and is a backward weak KAM solution of the Hamilton-Jacobi equation $H_{a}\left(x, u_{x}\right)=0$. (2) essentially says that the new L-O semigroup converges faster than the L-O semigroup. (3) implies that $u \in C\left(M, \mathbb{R}^{1}\right)$ is a backward weak KAM solution if and only if it is a fixed point of $\left\{\tilde{T}_{t}^{a}\right\}_{t \geq 0}$. 
Remark 4.6. Just as we mentioned earlier, for each $\tau \in[0,1]$ and each $u \in$ $C\left(M, \mathbb{R}^{1}\right)$, the uniform $\operatorname{limit}_{\lim _{n \rightarrow+\infty}} \tilde{T}_{n}^{a, \tau} u$ exists and

$$
\lim _{n \rightarrow+\infty} \tilde{T}_{n}^{a, \tau} u=\lim _{n \rightarrow+\infty} T_{n}^{a} u=\bar{u} .
$$

It can be proved by slight modifications of the proof of (1) in Proposition 4.4 .

Proof. First we prove (1). Assume by contradiction that there exist $\varepsilon_{0}>0, t_{n} \rightarrow$ $+\infty$ and $x_{n} \in M$ such that

$$
\left|\tilde{T}_{t_{n}}^{a} u\left(x_{n}\right)-\bar{u}\left(x_{n}\right)\right| \geq \varepsilon_{0} .
$$

From the compactness of $M$, without loss of generality we assume that $x_{n} \rightarrow x_{0}$, $n \rightarrow+\infty$. In view of the definition of $\tilde{T}_{t}^{a}$, there exist $\sigma_{n} \in\left[t_{n}, 2 t_{n}\right]$ such that

$$
\left|T_{\sigma_{n}}^{a} u\left(x_{n}\right)-\bar{u}\left(x_{n}\right)\right| \geq \varepsilon_{0} .
$$

Let $n \rightarrow+\infty$. Since $(\sigma, x) \mapsto T_{\sigma}^{a} u(x)$ is continuous, then we have

$$
\lim _{\sigma \rightarrow+\infty} T_{\sigma}^{a} u\left(x_{0}\right) \neq \bar{u}\left(x_{0}\right),
$$

which contradicts $\lim _{\sigma \rightarrow+\infty} T_{\sigma}^{a} u=\bar{u}$.

Next we show (2). For each $t \geq 0$ and each $x \in M$, there exists $t \leq \sigma_{x} \leq 2 t$ such that

$$
\left|\tilde{T}_{t}^{a} u(x)-\bar{u}(x)\right|=\left|T_{\sigma_{x}}^{a} u(x)-\bar{u}(x)\right| .
$$

Since $\bar{u}$ is a fixed point of $\left\{T_{t}^{a}\right\}_{t \geq 0}$, then we have $\left|T_{\sigma_{x}}^{a} u(x)-\bar{u}(x)\right|=\mid T_{\sigma_{x}}^{a} u(x)-$ $T_{\sigma_{x}}^{a} \bar{u}(x) \mid \leq\left\|T_{\sigma_{x}}^{a} u-T_{\sigma_{x}}^{a} \bar{u}\right\|_{\infty}=\left\|T_{\sigma_{x}-t}^{a} \circ T_{t}^{a} u-T_{\sigma_{x}-t}^{a} \circ T_{t}^{a} \bar{u}\right\|_{\infty} \leq\left\|T_{t}^{a} u-T_{t}^{a} \bar{u}\right\|_{\infty}=$ $\left\|T_{t}^{a} u-\bar{u}\right\|_{\infty}$, where we have used the non-expansiveness property of $T_{\sigma_{x}-t}^{a}$ (see [19, Corollary 4.4.4]). Hence (2) holds.

At last, we show (3). Suppose that $u$ is a fixed point of $\left\{T_{t}^{a}\right\}_{t \geq 0}$, i.e., $T_{t}^{a} u=u$, $\forall t \geq 0$. Then $\lim _{t \rightarrow+\infty} T_{t}^{a} u=u$. From (2) we have

$$
\left\|\tilde{T}_{t}^{a} u-u\right\|_{\infty} \leq\left\|T_{t}^{a} u-u\right\|_{\infty}=0, \quad \forall t \geq 0,
$$

which implies that $u$ is a fixed point of $\left\{\tilde{T}_{t}^{a}\right\}_{t>0}$. Suppose conversely that $u$ is a fixed point of $\left\{\tilde{T}_{t}^{a}\right\}_{t \geq 0}$. Then from (1) $\lim _{t \rightarrow+\infty} \tilde{T}_{t}^{a} u=u=\lim _{t \rightarrow+\infty} T_{t}^{a} u$. Hence $u$ is a backward weak KAM solution of $H_{a}\left(x, u_{x}\right)=0$ and a fixed point of $\left\{T_{t}^{a}\right\}_{t \geq 0}$.

4.2. Rates of convergence of the L-O semigroup and the new L-O semi-

group. Recall the $C^{2}$ positive definite and superlinear Lagrangian (1.6)

$$
L_{a}^{1}(x, v)=\frac{1}{2}\langle A(x)(v-\omega),(v-\omega)\rangle+f(x, v-\omega), \quad x \in \mathbb{T}^{n}, v \in \mathbb{R}^{n} .
$$

The conjugated Hamiltonian $H_{a}^{1}: \mathbb{T}^{n} \times \mathbb{R}^{n} \rightarrow \mathbb{R}^{1}$ of $L_{a}^{1}$ has the following form 


$$
H_{a}^{1}(x, p)=\langle\omega, p\rangle+\frac{1}{2}\left\langle A^{-1}(x) p, p\right\rangle+g(x, p),
$$

where $g(x, p)=O\left(\|p\|^{3}\right)$ as $p \rightarrow 0$. It is clear that $H_{a}^{1}(x, 0)=0$ and thus $w \equiv$ const. is a smooth viscosity solution of the corresponding Hamilton-Jacobi equation $H_{a}^{1}\left(x, u_{x}\right)=0$. In view of the Legendre transform,

$L_{a}^{1}(x, v)=L_{a}^{1}(x, v)-\left\langle w_{x}, v\right\rangle \geq-H_{a}^{1}\left(x, w_{x}\right)=-H_{a}^{1}(x, 0)=0, \quad \forall(x, v) \in \mathbb{T}^{n} \times \mathbb{R}^{n}$.

Furthermore, if $(x, v) \in \tilde{\mathcal{M}}_{0}=\cup_{x \in \mathbb{T}^{n}}(x, \omega)$, then $w_{x}=\frac{\partial L}{\partial v}(x, v)$ (see 19, Theorem 4.8.3]), from which we have

$$
L_{a}^{1}(x, v)=L_{a}^{1}(x, v)-\left\langle w_{x}, v\right\rangle=-H_{a}^{1}\left(x, w_{x}\right)=-H_{a}^{1}(x, 0)=0 .
$$

Hence

$$
L_{a}^{1} \geq 0, \quad \forall(x, v) \in \mathbb{T}^{n} \times \mathbb{R}^{n}
$$

and in particular,

$$
\left.L_{a}^{1}\right|_{\cup_{x \in \mathbb{T}^{n}}(x, \omega)}=0 .
$$

For each $u \in C\left(\mathbb{T}^{n}, \mathbb{R}^{1}\right)$, because of $c\left(L_{a}^{1}\right)=0$ we have $\lim _{t \rightarrow+\infty} T_{t}^{a} u=\bar{u}$. Note that both $w \equiv$ const. and $\bar{u}$ are viscosity solutions of $H_{a}^{1}\left(x, u_{x}\right)=0$. Hence $\bar{u} \equiv$ const. since the viscosity solution of $H_{a}^{1}\left(x, u_{x}\right)=0$ is unique up to constants when $\mathcal{A}_{0}=\mathbb{T}^{n}$ [27], where $\mathcal{A}_{0}$ is the projected Aubry set.

4.2.1. Rate of convergence of the L-O semigroup. We present here the proof of Theorem 1.5. For this, the following lemma is needed.

Lemma 4.7. For each $u \in C\left(\mathbb{T}^{n}, \mathbb{R}^{1}\right), \bar{u} \equiv \min _{x \in \mathbb{T}^{n}} u(x)$.

Proof. For any $x \in \mathbb{T}^{n}$, from the definition of $T_{t}^{a}$ we have

$$
\bar{u}(x)=\lim _{t \rightarrow+\infty} T_{t}^{a} u(x)=\lim _{t \rightarrow+\infty} \inf _{z \in \mathbb{T}^{n}}\left\{u(z)+\int_{0}^{t} L_{a}^{1}\left(\gamma_{z}, \dot{\gamma}_{z}\right) d s\right\},
$$

where $\gamma_{z}:[0, t] \rightarrow \mathbb{T}^{n}$ is a Tonelli minimizer with $\gamma_{z}(0)=z, \gamma_{z}(t)=x$. Since $L_{a}^{1} \geq 0$, then $\bar{u}(x) \geq \min _{z \in \mathbb{T}^{n}} u(z)$ and therefore it suffices to show that $\bar{u}(x) \leq$ $\min _{z \in \mathbb{T}^{n}} u(z)$.

Take $y \in \mathbb{T}^{n}$ with $u(y)=\min _{z \in \mathbb{T}^{n}} u(z)$. Consider the following two curves

$$
\gamma_{\omega}:[0, t] \rightarrow \mathbb{T}^{n}, s \mapsto \omega s+y
$$

and

$$
\gamma_{\omega^{\prime}}:[0, t] \rightarrow \mathbb{T}^{n}, s \mapsto \omega^{\prime} s+y
$$


with $\gamma_{\omega^{\prime}}(t)=x$, where $\omega^{\prime} \in \mathbb{S}^{n-1}$ and $t>0$. It is clear that $\gamma_{\omega^{\prime}}$ is a curve in $\mathbb{T}^{n}$ connecting $y$ and $x$. Let $\Delta=\gamma_{\omega^{\prime}}(t)-\gamma_{\omega}(t)=x-(\omega t+y)$. Then $\|\Delta\| \leq \frac{\sqrt{n}}{2}$ and $\dot{\gamma}_{\omega^{\prime}} \equiv \omega^{\prime}=\frac{\Delta}{t}+\omega$. Therefore, we have

$$
\begin{aligned}
T_{t}^{a} u(x) & \leq u\left(\gamma_{\omega^{\prime}}(0)\right)+\int_{0}^{t} L_{a}^{1}\left(\gamma_{\omega^{\prime}}, \dot{\gamma}_{\omega^{\prime}}\right) d s \\
& =u(y)+\int_{0}^{t}\left(\frac{1}{2}\left\langle A\left(\gamma_{\omega^{\prime}}\right)\left(\omega^{\prime}-\omega\right),\left(\omega^{\prime}-\omega\right)\right\rangle+f\left(\gamma_{\omega^{\prime}}, \omega^{\prime}-\omega\right)\right) d s \\
& =u(y)+\int_{0}^{t}\left(\frac{1}{2}\left\langle A\left(\gamma_{\omega^{\prime}}\right) \frac{\Delta}{t}, \frac{\Delta}{t}\right\rangle+f\left(\gamma_{\omega^{\prime}}, \frac{\Delta}{t}\right)\right) d s \\
& \leq u(y)+\frac{C}{t}+O\left(\frac{1}{t^{2}}\right),
\end{aligned}
$$

where $C$ is a constant, which depends only on $n$.

From the arguments above we know that for any $\varepsilon>0$, there exists $T>0$ such that for any $t>T$ there exists $\gamma_{\omega^{\prime}}:[0, t] \rightarrow \mathbb{T}^{n}$ with $\gamma_{\omega^{\prime}}(t)=x$, and

$$
T_{t}^{a} u(x) \leq u\left(\gamma_{\omega^{\prime}}(0)\right)+\int_{0}^{t} L_{a}^{1}\left(\gamma_{\omega^{\prime}}, \dot{\gamma}_{\omega^{\prime}}\right) d s \leq \min _{z \in \mathbb{T}^{n}} u(z)+\varepsilon .
$$

Hence $\bar{u}(x)=\lim _{t \rightarrow+\infty} T_{t}^{a} u(x) \leq \min _{z \in \mathbb{T}^{n}} u(z)$.

Proof of Theorem 1.5. In order to prove our result, it is sufficient to show that for each $u \in C\left(\mathbb{T}^{n}, \mathbb{R}^{1}\right)$, there exists a constant $K>0$ such that the following two inequalities hold.

$$
\begin{aligned}
& T_{t}^{a} u(x)-\bar{u}(x) \leq \frac{K}{t}, \quad \forall t>0, \forall x \in \mathbb{T}^{n} ; \\
& \bar{u}(x)-T_{t}^{a} u(x) \leq \frac{K}{t}, \quad \forall t>0, \forall x \in \mathbb{T}^{n} .
\end{aligned}
$$

Obviously, (I2) holds. In fact, for each $t>0$ and each $x \in \mathbb{T}^{n}$, from the definition of $T_{t}^{a}$ we have

$$
T_{t}^{a} u(x)=\inf _{z \in \mathbb{T}^{n}}\left\{u(z)+\int_{0}^{t} L_{a}^{1}\left(\gamma_{z}, \dot{\gamma}_{z}\right) d s\right\},
$$

where $\gamma_{z}:[0, t] \rightarrow \mathbb{T}^{n}$ is a Tonelli minimizer with $\gamma_{z}(0)=z, \gamma_{z}(t)=x$. In view of $L_{a}^{1} \geq 0$ and Lemma 4.7, we have

$$
T_{t}^{a} u(x)=\inf _{z \in \mathbb{T}^{n}}\left\{u(z)+\int_{0}^{t} L_{a}^{1}\left(\gamma_{z}, \dot{\gamma}_{z}\right) d s\right\} \geq \min _{z \in \mathbb{T}^{n}} u(z)=\bar{u}(x) .
$$

Thus $\bar{u}(x)-T_{t}^{a} u(x) \leq 0, \forall t>0, \forall x \in \mathbb{T}^{n}$ and (I2) holds.

Next we prove (I1). It suffices to show that there exists a constant $C>0$ such that for sufficiently large $t>0$, 


$$
T_{t}^{a} u(x)-\bar{u}(x) \leq \frac{C}{t}, \quad \forall x \in \mathbb{T}^{n},
$$

where $C$ depends only on $n$. In deed, since $(s, z) \mapsto T_{s} u(z)$ is continuous on $[0, \infty) \times \mathbb{T}^{n}$, if (4.5) holds, then there exists a constant $K>0$ such that

$$
T_{t}^{a} u(x)-\bar{u}(x) \leq \frac{K}{t}, \quad \forall t>0, \forall x \in \mathbb{T}^{n},
$$

where $K$ depends only on $n$ and $u$.

Take $y \in \mathbb{T}^{n}$ with $u(y)=\min _{z \in \mathbb{T}^{n}} u(z)$. Let us consider the following curve in $\mathbb{T}^{n}$

$$
\gamma_{\omega}:[0, t] \rightarrow \mathbb{T}^{n}, s \mapsto \omega s+y,
$$

where $t>0$. Then for each $x \in \mathbb{T}^{n}$, let

$$
\gamma_{\omega^{\prime}}:[0, t] \rightarrow \mathbb{T}^{n}, s \mapsto \omega^{\prime} s+y
$$

be a curve in $\mathbb{T}^{n}$ connecting $y$ and $x$, where $\omega^{\prime} \in \mathbb{S}^{n-1}$. Let $\Delta=\gamma_{\omega^{\prime}}(t)-\gamma_{\omega}(t)=$ $x-(\omega t+y)$. Then $\|\Delta\| \leq \frac{\sqrt{n}}{2}$ and $\dot{\gamma}_{\omega^{\prime}} \equiv \omega^{\prime}=\frac{\Delta}{t}+\omega$. Hence,

$$
\begin{aligned}
T_{t}^{a} u(x) & \leq u\left(\gamma_{\omega^{\prime}}(0)\right)+\int_{0}^{t} L_{a}^{1}\left(\gamma_{\omega^{\prime}}, \dot{\gamma}_{\omega^{\prime}}\right) d s \\
& =u(y)+\int_{0}^{t}\left(\frac{1}{2}\left\langle A\left(\gamma_{\omega^{\prime}}\right)\left(\omega^{\prime}-\omega\right),\left(\omega^{\prime}-\omega\right)\right\rangle+f\left(\gamma_{\omega^{\prime}}, \omega^{\prime}-\omega\right)\right) d s \\
& =u(y)+\int_{0}^{t}\left(\frac{1}{2}\left\langle A\left(\gamma_{\omega^{\prime}}\right) \frac{\Delta}{t}, \frac{\Delta}{t}\right\rangle+f\left(\gamma_{\omega^{\prime}}, \frac{\Delta}{t}\right)\right) d s \\
& \leq u(y)+\frac{C_{1}}{t}+O\left(\frac{1}{t^{2}}\right),
\end{aligned}
$$

where $C_{1}$ is a constant which depends only on $n$. From Lemma 4.7, we have $T_{t}^{a} u(x)-\bar{u}(x) \leq \frac{C}{t}$ for $t>0$ large enough, where $C$ is a constant which still depends only on $n$, i.e., (4.5) holds.

4.2.2. Rate of convergence of the new L-O semigroup. To complete the proof of Theorem 1.6, we review preliminaries on the ergodization rate for linear flows on the torus $\mathbb{T}^{n}$, i.e., the rate at which the image of a point fills the torus when subjected to linear flows. There is a direct relationship between the rate of convergence of the new L-O semigroup and the ergodization rate for linear flows on the torus $\mathbb{T}^{n}$. Let us recall the following result of Dumas' 10] concerning the estimate of ergodization time.

For each $t \in \mathbb{R}^{1}$ and each $\omega \in \mathbb{S}^{n-1}$, consider the one-parameter family of translation maps $\omega_{t}: \mathbb{T}^{n} \rightarrow \mathbb{T}^{n}, x \mapsto x+\omega t$. A rectilinear orbit of $\mathbb{T}^{n}$ with direction vector $\omega$ and initial condition $x$ is defined as the image of $x$ under the linear flow $\omega_{t}$ over some closed interval $\left[t_{0}, t_{1}\right] \subset \mathbb{R}^{1}$, i.e., 


$$
\bigcup_{t_{0} \leq t \leq t_{1}} \omega_{t}(x)
$$

Given $R>0$, the direction vector $\omega \in \mathbb{S}^{n-1}$ is said to ergodize $\mathbb{T}^{n}$ to within $R$ after time $T$ if

$$
\bigcup_{0 \leq t \leq T} \omega_{t}\left(B_{R}(x)\right)=\mathbb{T}^{n}
$$

for all $x \in \mathbb{T}^{n}$.

As defined in the Introduction, for $\rho>n-1$ and $\alpha>0$,

$$
\mathcal{D}(\rho, \alpha)=\left\{\beta \in \mathbb{S}^{n-1}||\langle\beta, k\rangle \mid>\frac{\alpha}{|k|^{\rho}}, \forall k \in \mathbb{Z}^{n} \backslash\{0\}\right\},
$$

whose elements can not be approximated by rationals too rapidly.

Theorem 4.8 (Dumas [10]). Let $0<R \leq 1$. Given any highly nonresonant direction vector $\omega \in \mathcal{D}(\rho, \alpha)$, rectilinear orbits of $\mathbb{T}^{n}$ with direction vector $\omega$ will ergodize $\mathbb{T}^{n}$ to within $R$ after time $T$, where

is independent of $\omega$.

$$
T=\frac{2\left\|V_{*}\right\|_{\triangle}}{\alpha \pi R^{\rho+n / 2}}
$$

Remark 4.9. The constant $\left\|V_{*}\right\|_{\triangle}$ is a Sobolev norm of a certain "smoothest test function" and it depends only on $n$ and $\rho$. See [10] for complete details.

We are now in a position to give the proof of Theorem 1.6. Proof of Theorem 1.6. Our purpose is to show that for each $u \in C\left(\mathbb{T}^{n}, \mathbb{R}^{1}\right)$, there exists a constant $\tilde{K}>0$ such that the following two inequalities hold.

$$
\begin{aligned}
& \tilde{T}_{t}^{a} u(x)-\bar{u}(x) \leq \tilde{K} t^{-\left(1+\frac{4}{2 \rho+n}\right)}, \quad \forall t>0, \forall x \in \mathbb{T}^{n} \\
& \bar{u}(x)-\tilde{T}_{t}^{a} u(x) \leq \tilde{K} t^{-\left(1+\frac{4}{2 \rho+n}\right)}, \quad \forall t>0, \forall x \in \mathbb{T}^{n} .
\end{aligned}
$$

First we show (I4). For each $t>0$ and each $x \in \mathbb{T}^{n}$, by the definition of $\tilde{T}_{t}^{a}$ we have

$$
\tilde{T}_{t}^{a} u(x)=\inf _{t \leq \sigma \leq 2 t} \inf _{z \in \mathbb{T}^{n}}\left\{u(z)+\int_{0}^{\sigma} L_{a}^{1}\left(\gamma_{z}, \dot{\gamma}_{z}\right) d s\right\}
$$

where $\gamma_{z}:[0, \sigma] \rightarrow \mathbb{T}^{n}$ is a Tonelli minimizer with $\gamma_{z}(0)=z, \gamma_{z}(\sigma)=x$. In view of $L_{a}^{1} \geq 0$ and Lemma 4.7, we have

$$
\tilde{T}_{t}^{a} u(x)=\inf _{t \leq \sigma \leq 2 t} \inf _{z \in \mathbb{T}^{n}}\left\{u(z)+\int_{0}^{\sigma} L_{a}^{1}\left(\gamma_{z}, \dot{\gamma}_{z}\right) d s\right\} \geq \min _{z \in \mathbb{T}^{n}} u(z)=\bar{u}(x) .
$$

Thus $\bar{u}(x)-\tilde{T}_{t}^{a} u(x) \leq 0, \forall t>0, \forall x \in \mathbb{T}^{n}$, i.e., (I4) holds. 
Then it remains to show (I3). When $R=1$, according to Theorem 4.8 the ergodization time $T=\frac{2\left\|V_{*}\right\|_{\Delta}}{\alpha \pi}$. For any $t \geq T$, let $R_{t}=\sqrt[\rho+n / 2]{\frac{2\left\|V_{*}\right\|_{\Delta}}{\alpha \pi t}}$. Then $0<R_{t} \leq 1$.

Take $y \in \mathbb{T}^{n}$ with $u(y)=\min _{z \in \mathbb{T}^{n}} u(z)$. Let $y_{t}=\omega_{t}(y)=\omega t+y$. For $R_{t}$ defined above, since $\omega \in \mathcal{D}(\rho, \alpha)$, then from Theorem 4.8 and (4.6) we have

$$
\bigcup_{0 \leq \varsigma \leq t} \omega_{\varsigma}\left(B_{R_{t}}\left(y_{t}\right)\right)=\mathbb{T}^{n}
$$

Therefore, for each $x \in \mathbb{T}^{n}$, there exists $0 \leq \varsigma^{\prime} \leq t$ such that $d_{\mathbb{T}^{n}}\left(\omega_{\varsigma^{\prime}}\left(y_{t}\right), x\right) \leq R_{t}$, i.e., $d_{\mathbb{T}^{n}}\left(\omega\left(t+\varsigma^{\prime}\right)+y, x\right) \leq R_{t}$. Equivalently this means that there exists $t \leq \sigma^{\prime} \leq 2 t$ such that

$$
d_{\mathbb{T}^{n}}\left(\omega \sigma^{\prime}+y, x\right) \leq R_{t}
$$

where $\sigma^{\prime}=t+\varsigma^{\prime}$. Consider the following curve in $\mathbb{T}^{n}$

$$
\gamma_{\omega^{\prime}}:\left[0, \sigma^{\prime}\right] \rightarrow \mathbb{T}^{n}, s \mapsto \omega^{\prime} s+y
$$

with $\gamma_{\omega^{\prime}}\left(\sigma^{\prime}\right)=x$, where $\omega^{\prime} \in \mathbb{S}^{n-1}$. It is clear that $\gamma_{\omega^{\prime}}$ connects $y$ and $x$. Let $\Delta=\gamma_{\omega^{\prime}}\left(\sigma^{\prime}\right)-\omega_{\sigma^{\prime}}(y)=x-\left(\omega \sigma^{\prime}+y\right)$. Then $\|\Delta\|=d_{\mathbb{T}^{n}}\left(x, \omega \sigma^{\prime}+y\right) \leq R_{t}$ and $\dot{\gamma}_{\omega^{\prime}} \equiv \omega^{\prime}=\frac{\Delta}{\sigma^{\prime}}+\omega$. Hence we have

$$
\begin{aligned}
\tilde{T}_{t}^{a} u(x)-\bar{u}(x) & \leq u\left(\gamma_{\omega^{\prime}}(0)\right)+\int_{0}^{\sigma^{\prime}} L_{a}^{1}\left(\gamma_{\omega^{\prime}}, \dot{\gamma}_{\omega^{\prime}}\right) d s-\bar{u}(x) \\
& =\int_{0}^{\sigma^{\prime}}\left(\frac{1}{2}\left\langle A\left(\gamma_{\omega^{\prime}}\right)\left(\omega^{\prime}-\omega\right),\left(\omega^{\prime}-\omega\right)\right\rangle+f\left(\gamma_{\omega^{\prime}}, \omega^{\prime}-\omega\right)\right) d s \\
& \leq \frac{C R_{t}^{2}}{t}
\end{aligned}
$$

for sufficiently large $t>0$ and some constant $C>0$. Since $R_{t}^{2}=\left(\frac{2\left\|V_{*}\right\| \Delta}{\alpha \pi t}\right)^{\frac{2}{\rho+n / 2}}$, then for $t>0$ large enough we have

$$
\tilde{T}_{t}^{a} u(x)-\bar{u}(x) \leq C_{1} t^{-\left(1+\frac{4}{2 \rho+n}\right)}, \quad \forall x \in \mathbb{T}^{n},
$$

where $C_{1}$ is a constant which depends only on $n, \rho$ and $\alpha$. From (5) of Proposition 4.2, $(s, z) \mapsto \tilde{T}_{s}^{a} u(z)$ is continuous on $[0, \infty) \times \mathbb{T}^{n}$. Hence there exists a constant $\tilde{K}>0$ such that

$$
\tilde{T}_{t}^{a} u(x)-\bar{u}(x) \leq \tilde{K} t^{-\left(1+\frac{4}{2 \rho+n}\right)}, \quad \forall t>0, \forall x \in \mathbb{T}^{n},
$$

where $\tilde{K}$ depends only on $n, \rho, \alpha$ and $u$, i.e., (I3) holds. 


\subsubsection{An example.}

Example 4.10. Consider the following integrable $C^{2}$ Lagrangian

$$
\bar{L}_{a}^{1}(x, v)=\frac{1}{2}\langle v-\omega, v-\omega\rangle, \quad x \in \mathbb{T}^{n}, v \in \mathbb{R}^{n}, \omega \in \mathbb{S}^{n-1} .
$$

It is easy to see that $\bar{L}_{a}^{1}$ is a special case of $L_{a}^{1}$. For $\bar{L}_{a}^{1}$, we show that there exist $u \in C\left(\mathbb{T}^{n}, \mathbb{R}^{1}\right), x^{0} \in \mathbb{T}^{n}$ and $t_{m} \rightarrow+\infty$ as $m \rightarrow+\infty$ such that

$$
\left|T_{t_{m}}^{a} u\left(x^{0}\right)-\bar{u}\left(x^{0}\right)\right|=O\left(\frac{1}{t_{m}}\right), \quad m \rightarrow+\infty,
$$

which implies that the result of Theorem 1.5 is sharp in the sense of order.

Recall the universal covering projection $\pi: \mathbb{R}^{n} \rightarrow \mathbb{T}^{n}$. Let $x^{0} \in \mathbb{T}^{n}$ such that each point $\tilde{x}^{0} \in \mathbb{R}^{n}$ in the fiber over $x^{0}\left(\pi \tilde{x}^{0}=x^{0}\right)$ is the center of each fundamental domain in $\mathbb{R}^{n}$. Define a continuous function on $\mathbb{R}^{n}$ as follows: for $\tilde{x} \in \mathbb{R}^{n}$

$$
\tilde{u}(\tilde{x})= \begin{cases}\delta-\left\|\tilde{x}-\tilde{x}^{0}\right\|, & \left\|\tilde{x}-\tilde{x}^{0}\right\| \leq \delta, \\ 0, & \text { otherwise }\end{cases}
$$

where $0<\delta<\frac{1}{2}$. We then define a continuous function on $\mathbb{T}^{n}$ as $u(x)=\tilde{u}(\tilde{x})$ for all $x \in \mathbb{T}^{n}$, where $\tilde{x}$ is an arbitrary point in the fiber over $x$. Thus, from Lemma 4.7. $\bar{u} \equiv \min _{x \in \mathbb{T}^{n}} u(x)=0$.

Now fix a point $\tilde{x}_{0}^{0}$ in the fiber over $x^{0}$. Then there exist $\left\{\tilde{x}_{m}^{0}\right\}_{m=1}^{+\infty}$ in the fiber over $x^{0}$ and $t_{m} \rightarrow+\infty$ as $m \rightarrow+\infty$ such that $\left\|\left(\tilde{x}_{m}^{0}-\omega t_{m}\right)-\tilde{x}_{0}^{0}\right\| \leq \frac{\delta}{2}$. Let $\tilde{z}_{m}=\tilde{x}_{m}^{0}-\omega t_{m}$. Then $\left\|\tilde{z}_{m}-\tilde{x}_{0}^{0}\right\| \leq \frac{\delta}{2}$. For each $t_{m}$ there exists $y_{m} \in \mathbb{T}^{n}$ such that

$$
T_{t_{m}}^{a} u\left(x^{0}\right)=u\left(y_{m}\right)+\int_{0}^{t_{m}} \bar{L}_{a}^{1}\left(\gamma_{y_{m}}, \dot{\gamma}_{y_{m}}\right) d s
$$

where $\gamma_{y_{m}}:\left[0, t_{m}\right] \rightarrow \mathbb{T}^{n}$ is a Tonelli minimizer with $\gamma_{y_{m}}(0)=y_{m}, \gamma_{y_{m}}\left(t_{m}\right)=x^{0}$. In view of the lifting property of the covering projection, there is a unique curve $\tilde{\gamma}_{y_{m}}:\left[0, t_{m}\right] \rightarrow \mathbb{R}^{n}$ with $\pi \tilde{\gamma}_{y_{m}}=\gamma_{y_{m}}$ and $\tilde{\gamma}_{y_{m}}\left(t_{m}\right)=\tilde{x}_{m}^{0}$. Set $\tilde{y}_{m}=\tilde{\gamma}_{y_{m}}(0)$. Then $\pi \tilde{y}_{m}=y_{m}$. Moreover, $\tilde{\gamma}_{y_{m}}$ has the following form

$$
\tilde{\gamma}_{y_{m}}(s)=\omega^{\prime} s+\tilde{y}_{m}, \quad s \in\left[0, t_{m}\right]
$$

where $\omega^{\prime} \in \mathbb{S}^{n-1}$. It is clear that $\tilde{\gamma}_{y_{m}}(0)=\tilde{y}_{m}$ and $\tilde{y}_{m}=\tilde{x}_{m}^{0}-\omega^{\prime} t_{m}$.

If $\left\|\tilde{y}_{m}-\tilde{z}_{m}\right\| \leq \frac{\delta}{4}$, then from $\left\|\tilde{z}_{m}-\tilde{x}_{0}^{0}\right\| \leq \frac{\delta}{2}$ we have $\left\|\tilde{y}_{m}-\tilde{x}_{0}^{0}\right\| \leq \frac{3 \delta}{4}$. Hence,

$$
\begin{aligned}
T_{t_{m}}^{a} u\left(x^{0}\right) & =u\left(y_{m}\right)+\int_{0}^{t_{m}} \bar{L}_{a}^{1}\left(\gamma_{y_{m}}, \dot{\gamma}_{y_{m}}\right) d s \\
& \geq \tilde{u}\left(\tilde{y}_{m}\right) \geq \delta-\frac{3 \delta}{4}=\frac{\delta}{4} .
\end{aligned}
$$

From (4.7), we may deduce that there can only be a finite number of $\tilde{y}_{m}$ 's such that $\left\|\tilde{y}_{m}-\tilde{z}_{m}\right\| \leq \frac{\delta}{4}$. For, otherwise, there would be $\left\{t_{m_{i}}\right\}_{i=1}^{+\infty}$ and $\left\{\tilde{y}_{m_{i}}\right\}_{i=1}^{+\infty}$ such that 


$$
T_{t_{m_{i}}}^{a} u\left(x^{0}\right) \geq \frac{\delta}{4}, \quad i=1,2, \cdots,
$$

which contradicts $\lim _{i \rightarrow+\infty} T_{t_{m_{i}}}^{a} u\left(x^{0}\right)=\bar{u}\left(x^{0}\right)=0$.

For $\tilde{y}_{m}$ with $\left\|\tilde{y}_{m}-\tilde{z}_{m}\right\|>\frac{\delta}{4}$, we have

$$
\frac{\delta}{4}<\left\|\tilde{y}_{m}-\tilde{z}_{m}\right\|=\left\|\tilde{x}_{m}^{0}-\omega^{\prime} t_{m}-\left(\tilde{x}_{m}^{0}-\omega t_{m}\right)\right\|=\left\|\omega-\omega^{\prime}\right\| t_{m} .
$$

Thus,

$$
\begin{aligned}
T_{t_{m}}^{a} u\left(x^{0}\right) & =u\left(y_{m}\right)+\int_{0}^{t_{m}} \bar{L}_{a}^{1}\left(\gamma_{y_{m}}, \dot{\gamma}_{y_{m}}\right) d s \\
& \geq \frac{1}{2} t_{m}\left\|\omega-\omega^{\prime}\right\|^{2}=\frac{1}{2} \frac{t_{m}^{2}\left\|\omega-\omega^{\prime}\right\|^{2}}{t_{m}} \geq \frac{\delta^{2}}{32 t_{m}} .
\end{aligned}
$$

Therefore, from (4.8) and Theorem 1.5 we have

$$
\left|T_{t_{m}}^{a} u\left(x^{0}\right)-\bar{u}\left(x^{0}\right)\right|=\left|T_{t_{m}}^{a} u\left(x^{0}\right)\right|=O\left(\frac{1}{t_{m}}\right), \quad m \rightarrow+\infty .
$$

\section{REFERENCES}

[1] M. Arnaud, Pseudographs and the Lax-Oleinik semi-group: a geometric and dynamical interpretation, to appear in Nonlinearity.

[2] M. Arnaud, The link between the shape of the Aubry-Mather sets and their Lyapunov exponents, arXiv:0902.3266 1 .

[3] P. Bernard, Connecting orbits of time dependent Lagrangian systems, Ann. Inst. Fourier (Grenoble) 52 (2002), 1533-1568.

[4] P. Bernard, J.-M. Roquejoffre, Convergence to time-periodic solutions in time-periodic Hamilton-Jacobi equations on the circle, Comm. Partial Differential Equations 29 (2004), 457-469.

[5] P. Bernard, The dynamics of pseudographs in convex Hamiltonian systems, J. Amer. Math. Soc. 21 (2008), 615-669.

[6] P. Bernard, G. Contreras, A generic property of families of Lagrangian systems. Ann. of Math. (2) 167 (2008), 1099-1108.

[7] G. Contreras, R. Iturriaga, Global Minimizers of Autonomous Lagrangians, $22^{\circ}$ Colóuio Brasileiro de Matemática. Instituto de Matemática Pura e Aplicada (IMPA), Rio de Janeiro, 1999.

[8] G. Contreras, Action potential and weak KAM solutions, Calc. Var. Partial Differential Equations 13 (2001), 427-458.

[9] G. Contreras, R. Iturriaga and H. Sánchez Morgado, Weak solutions of the Hamilton-Jacobi equation for Time Periodic Lagrangians, preprint.

[10] H. Dumas, Ergodization rates for linear flow on the torus, J. Dynam. Differential Equations 3 (1991), 593-610.

[11] L. Evans, A survey of partial differential equations methods in weak KAM theory, Comm. Pure Appl. Math. 57 (2004), 445-480.

[12] L. Evans, Weak KAM theory and partial differential equations. Calculus of variations and nonlinear partial differential equations, 123-154. Lecture Notes in Math., 1927, Springer, Berlin, 2008. 
[13] L. Evans, Further PDE methods for weak KAM theory, Calc. Var. Partial Differential Equations 35 (2009), 435-462.

[14] A. Fathi, Théorème KAM faible et théorie de Mather sur les systèmes lagrangiens, C. R. Acad. Sci. Paris Sér. I Math. 324 (1997), 1043-1046.

[15] A. Fathi, Solutions KAM faibles conjuguées et barrières de Peierls, C. R. Acad. Sci. Paris Sér. I Math. 325 (1997), 649-652.

[16] A. Fathi, Orbites hétéroclines et ensemble de Peierls, C. R. Acad. Sci. Paris Sér. I Math. 326 (1998), 1213-1216.

[17] A. Fathi, Sur la convergence du semi-groupe de Lax-Oleinik, C. R. Acad. Sci. Paris Sér. I Math. 327 (1998), 267-270.

[18] A. Fathi, J. Mather, Failure of convergence of the Lax-Oleinik semi-group in the time-periodic case, Bull. Soc. Math. France 128 (2000), 473-483.

[19] A. Fathi, Weak KAM Theorems in Lagrangian Dynamics, seventh preliminary version, Pisa, 2005.

[20] D. Gomes, Generalized Mather problem and selection principles for viscosity solutions and Mather measures, Adv. Calc. Var. 1 (2008), 291-307.

[21] D. Gomes, R. Iturriaga, H. Sánchez-Morgado and Y. Yu, Mather measures selected by an approximation scheme, Proc. Amer. Math. Soc. 138 (2010), 3591-3601.

[22] E. Hopf, The partial differential equation $u_{t}+u u_{x}=\mu u_{x x}$, Comm. Pure Appl. Math. 3 (1950), 201-230.

[23] R. Iturriaga, H. Sánchez-Morgado, Hyperbolicity and exponential convergence of the LaxOleinik semigroup, J. Differential Equations 246 (2009) 1744-1753.

[24] V. Kaloshin, Mather theory, weak KAM theory, and viscosity solutions of Hamilton-Jacobi PDE's, EQUADIFF 2003, 39-48, World Sci. Publ., Hackensack, NJ, 2005.

[25] M. Keller-Ressel, Intuitive Introduction to Operator Semi-Groups, Technische Universität Wien, 2006.

[26] P. Lax, Hyperbolic systems of conservation laws, Comm. Pure Appl. Math. 10 (1957), 537566.

[27] Z. Liang, J. Yan and Y. Yi, Viscous stability of quasi-periodic Lagrangian tori, preprint.

[28] P. Lions, Generalized Solutions of Hamilton-Jacobi Equations, Research Notes in Mathematics, London, Pitman Publishing, 1982.

[29] P. Lions, G. Papanicolaou and S. Varadhan, Homogenization of Hamilton-Jacobi equations, unpublished, circa, 1988.

[30] R. Mañé, On the minimizing measures of Lagrangian dynamical systems, Nonlinearity 5 (1992), 623-638.

[31] R. Mañé, Generic properties and problems of minimizing measures of Lagrangian systems, Nonlinearity 9 (1996), 273-310.

[32] R. Mañé, Lagrangian flows: the dynamics of globally minimizing orbits, Bol. Soc. Brasil. Mat. (N.S.) 28 (1997), 141-153.

[33] J. Mather, Action minimizing invariant measures for positive definite Lagrangian systems, Math. Z. 207 (1991), 169-207.

[34] J. Mather, Variational construction of connecting orbits, Ann. Inst. Fourier (Grenoble) 43 (1993), 1349-1386.

[35] J. Mather, G. Forni, Action minimizing orbits in Hamiltonian systems. Transition to chaos in classical and quantum mechanics (Montecatini Terme, 1991), 92-186. Lecture Notes in Math., 1589, Springer, Berlin, 1994.

[36] O. Oleinik, Discontinuous solutions of nonlinear differential equations, Uspekhi Mat. Nauk (N.S.) 12 (1957), 3-73. 
[37] A. Sorrentino, Lecture Notes on Mather's Theory for Lagrangian Systems, preprint, 2010.

[38] K. Wang, J. Yan, The rate of convergence of the Lax-Oleinik semigroup-Degenerate critical point case, to appear in Sci. China Math.

1 School of Mathematical Sciences and Key Lab of Mathematics for Nonlinear Science, Fudan University, Shanghai 200433, China

2 College of Mathematics, Jilin University, Changchun 130012, China

E-mail address: kaizhiwang@163.com; yanjun@fudan.edu.cn 